\title{
Milestones on the Road to Higher Resolution, Quantitative, and Functional Ultrasonic Imaging
}

\author{
S. LORI BRIDAL, MEMBER, IEEE, JEAN-MICHEL CORREAS, AMENA SAÏED, AND \\ PASCAL LAUGIER
}

\author{
Invited Paper
}

\begin{abstract}
Ultrasonography is evolving rapidly with important recent advances in high-density transducer arrays, one-and-one-half-dimensional transducers, broad-band transducers, increased scanner bandwidth, and more sophisticated image-formation routines. Technical advances have clearly improved accuracy of image readings, heightened contrast and resolution, reduced noise, and reduced image slice thickness. It is within this fertile environment that very high-frequency ultrasound, harmonic imaging, and ultrasound contrast agents have emerged. Clinical applications of ultrasonography have also been extended to new fields, such as skeletal status assessment, which have long been considered beyond ultrasound's reach, and over the past 15 years, quantitative ultrasound bone densitometry has become an important part of the armamentarium for osteoporosis diagnosis. The state of these innovations, their contributions to diagnostic and monitoring capabilities, as well as the new applications they bring into reach will be discussed. We will explore several applications currently under development including ultrasound biomicroscopy (eye, skin, small animals), quantitative perfusion assessment, and pathology evaluation. Thus, ongoing research has not only significantly added to diagnostic ultrasound's existing capabilities, but also promises to further broaden the range of its clinical and biological applications.
\end{abstract}

Keywords-Acoustic imaging, biomedical imaging, biomicroscopy, bone densitometry, harmonic analysis, ultrasound contrast agents (USCAs).
Manuscript received July 15, 2002; revised March 17, 2003.

S. L. Bridal, A. Saïed, and P. Laugier are with the Laboratoire d'Imagerie Paramétrique, Unité Mixte de Recherche (UMR) 7623, Centre National de la Recherche Scientifique (CNRS), University of Paris VI, 75006 Paris, France (e-mail: bridal@lip.bhdc.jussieu.fr; saied@lip.bhdc.jussieu.fr; laugier@lip.bhdc.jussieu.fr).

J.-M. Correas is with the Laboratoire d'Imagerie Paramétrique, Service de Radiologie Adulte, Hôpital Necker, Unité Mixte de Recherche (UMR) 7623, Centre National de la Recherche Scientifique (CNRS), University of Paris VI, 75006, Paris, France (e-mail: jean-michel.correas@ nck.ap-hop-paris.fr)

Digital Object Identifier 10.1109/JPROC.2003.817879

\section{INTRODUCTION}

Diagnostic ultrasound is used in almost all medical fields and is recognized as an increasingly important modality in a variety of clinical situations. This mode of imaging is evolving rapidly with important advances in transducer technology and more sophisticated image-formation routines. In addition, ultrasound equipment remains less expensive than all but certain conventional X-ray systems and is much less costly to purchase, operate, and maintain than $\mathrm{X}$-ray computed tomography (CT) or magnetic resonance imaging (MRI). Thus, clinical usefulness, recent technical advances, and continued affordability have contributed to make ultrasound more widely used and available today than ever before.

Engineering advances over the last decade have clearly improved accuracy of image readings, heightened contrast and resolution, reduced noise, and opened the way to new clinical applications. Recent advances include the introduction of high-density transducer arrays, one-and-onehalf-dimensional (1.5-D) transducers, enhanced bandwidth transducers, high-frequency transducer arrays, and progressive elimination of analog electronics. The super-wide-band highly sensitive high-density imaging transducer arrays provide greatly improved image quality. This has been further enhanced by the introduction of digital technology platforms that include digital beamformers and assure high dynamic ranges on the order of $120-140 \mathrm{~dB}$, as well as by the introduction of a myriad of approaches for electronically multifocal focusing and aperture control. Sophisticated image-formation routines, such as extended field-of-view or spatial compounding, have brought additional improvements in image quality. On another front, the frequency range for imaging has been pushed relentlessly higher. Specialized applications such as intraoperative imaging or breast imaging are now routinely performed at $12-15 \mathrm{MHz}$.

Several new and advanced ultrasound systems are being developed in laboratories or evaluated in clinical trials. 
Transducer miniaturization continues to increase the capabilities of intracavitary, intraurethral, or intravascular ultrasound (IVUS) investigation and is bringing ultrasound to the operating room for intraoperative application. Three-dimensional (3-D) ultrasound is also coming to maturity [1]. While 3-D has yet to reach its full potential in real-time imaging, many devices generate volumetric images in less than $1 \mathrm{~min}$, opening the door to new diagnostic opportunities. In obstetrics, imaging of the fetus in 3-D may permit a better definition of several congenital anomalies by allowing simultaneous viewing from multiple angles [2]. 3-D ultrasound diagnostic imaging is also well suited for precise volume measurements. This is important in oncology where planning of therapy and follow-up depends largely on accurate volumetry. Prostate ultrasound is another domain where 3-D ultrasound offers a promising new research tool that may prove useful to correctly identify the location, size, and, with the addition of 3-D power Doppler, the type of tumor [3], [4]. Similarly, fetal weight estimation can have a big impact on planning and decision making concerning both very small and very large fetuses. Currently, most 3-D imaging systems use arrays to generate two-dimensional (2-D) B-scans and obtain the third dimension by mechanical movement. This method is too slow for imaging moving structures such as the heart. The future development of real time 3-D heart imaging [5], [6] will require the design of 2-D transducer arrays.

In addition to the contributions brought by system-based improvements, new signal acquisition and analysis techniques are changing the face of ultrasound. Notably, the real-time nature of ultrasound has been exploited to detect tissue deformations induced by external stresses or natural vibrations within the body. Such "ultrasonic palpitation" of tissues, referred to as ultrasonic elastography, has generated an extremely active field of research, which is discussed in detail in another paper in this issue. Important advances have also been made in Doppler analysis and display. In particular, the detection of blood flow has been improved with higher sensitivity and higher spatial and temporal resolution. New modalities were recently introduced, such as directional power Doppler imaging, color dynamic flow, and B-flow imaging. Other major innovations have been described in recent years that may presage a revolution almost as great as the advent of real time or color Doppler imaging. These are contrast agents, ultrasound harmonic imaging, and very high-frequency ultrasound (also referred to as ultrasound biomicroscopy). New research in these domains appears in the literature every month.

The list of biological applications for diagnostic ultrasound also continues to lengthen. These include, for example, sonography of superficial structures (skin, musculoskeletal, nerves, breast, scrotum) [7]-[11], evaluation of arthritic diseases [12], ultrasound bone densitometry [13], and small animal imaging [14]. The new frontiers for ultrasound are literally extending to astronomical limits. An ultrasound scanner or a bone densitometer is to be included onboard of orbital stations to study the response of the cardiovascular system or skeleton to long-term weightlessness exposure [15]. Assessment of skeletal status with the goal of fracture risk prediction is a clinical field where quantitative ultrasound (QUS) has played a growing role in recent years. The significant growth in the QUS industry, with several important technical innovations, has been based on the affordability of the technology and the potential of elastic waves to probe multiple bone properties such as bone density, microarchitecture, and elasticity. The success of QUS technology in the field of clinical bone densitometry can be attributed to the extending availability of ultrasonic equipment, which provides equivalent fracture risk assessment compared to conventional X-ray absorptiometric techniques.

Faced with the plethora of innovations contributing to the recent expansion of diagnostic ultrasound and the multitude of clinical applications, we have chosen to concentrate on three rapidly developing domains of ultrasonic imaging, i.e., ultrasound contrast imaging, ultrasound biomicroscopy, and QUS bone densitometry. Ultrasound contrast imaging provides a good example of how the introduction of a new type of imaging target not only improved image contrast, but also inspired fundamental modifications in imaging modes and provided access to functional information that could not previously be obtained with ultrasound. Ultrasound biomicroscopy has driven development of high-frequency transducer technology and has opened up entirely new fields of investigation such as dermatosonography, IVUS, and ultrasonic imaging of the laboratory mouse. The third domain to be presented, i.e., ultrasound bone densitometry, has demonstrated that ultrasound is not limited to imaging of soft tissues after all and that it can provide truly quantitative diagnostic information. The state of these innovations, their contributions to diagnostic and monitoring capabilities, as well as the new applications they bring into reach will be discussed.

\section{ULTRASOUND CONTRAST IMAGING}

\section{A. Introduction}

Ultrasound contrast agents (USCAs), consisting of strongly reflecting tiny gas bubbles injected in solution intravenously, improve image quality by increasing the intensity of backscattered echoes from blood-filled regions or vascularized tissues. The first injectable microbubbles were commercialized during the late 1990s after their clinical utility in the enhancement of weak-echo signals both in B-mode (greyscale imaging of cardiac cavities) and in spectral or color Doppler sonography had been proven [16]. However, first-generation microbubble agents were not capable of crossing the pulmonary capillary bed due to pressure sensitivity and poor stabilization providing a very short half-life of several hundred milliseconds [17]. Furthermore, many agents were easily destroyed at acoustic imaging pressures. As initial bubble-based contrast agents appeared too unstable, research was oriented toward nongaseous contrast such as colloidal suspensions, emulsions, or aqueous solutions. However, these agents have not been able to offer the levels of contrast enhancement and safety profile 
Table 1

Description of Available USCAs

\begin{tabular}{|c|c|c|c|}
\hline Agent & Stabilization & Gas & Vendor \\
\hline Echovist ${ }^{\circledR}$ & Galactose matrix & Air & Schering SA \\
\hline Albunex ${ }^{\circledR}$ & Albumin & Air & $\begin{array}{l}\text { Molecular Biotechnology Inc., } \\
\text { Mallinckrodt }\end{array}$ \\
\hline Levovist ${ }^{\circledR}$ & $\begin{array}{l}\text { Galactose matrix, palmitic } \\
\text { acid }\end{array}$ & Air & Schering SA \\
\hline Optison® & Albumin & Octafluoropropane & $\begin{array}{l}\text { Molecular Biotechnology Inc., } \\
\text { Mallinckrodt }\end{array}$ \\
\hline SonoVue ${ }^{\mathrm{TM}}$ & $\begin{array}{l}\text { Surfactants, } \\
\text { phospholipides, palmitic } \\
\text { acid }\end{array}$ & Sulfur hexafluoride & Bracco \\
\hline Definity $^{\mathrm{TM}}$ & $\begin{array}{l}\text { Lipid bilayers } \\
\text { (dipalmitoylphosphatidylch } \\
\text { oline) }\end{array}$ & Octafluoropropane & DuPont Pharmaceutical \\
\hline $\begin{array}{l}\text { Imagent/ } \\
\text { Imavist }{ }^{\circledR}\end{array}$ & Surfactants & Perfluorohexane & Alliance Pharmaceuticals, Schering SA \\
\hline Sonozoid ${ }^{T M}$ & Surfactants & Perfluorobutane & Amersham Health \\
\hline Sonovist ${ }^{\circledR}$ & Cyanoacrylate & Air & Schering SA \\
\hline
\end{tabular}

obtainable with gas microbubbles [18], [19]. Stabilized microbubbles, consisting of encapsulated low-solubility gases, have recently been introduced in clinical practice. With greater stability and more reliable echogenicity, these agents have inspired the development of real-time contrast-specific imaging modalities and have been a critical catalyst in the advent of harmonic imaging during the second half of the 1990s.

Thus, after a relatively long period of development, USCAs are finally beginning to enter into routine clinical practice where they are extending the usefulness of ultrasound imaging. This major evolution can be attributed to a strong improvement in their persistence and efficacy, as well as to the development of contrast-specific imaging techniques. Their clinical potential is being further explored with the introduction of new agents presenting a strong acoustic response even at low incident acoustic power.

\section{B. Development of USCAs}

Recently commercialized USCAs based on stabilized microbubbles present both an excellent safety profile and a strong acoustic response, including a harmonic response exploitable for nonlinear imaging. They are able to pass through the pulmonary capillary bed after a peripheral intravenous injection since their diameter is much smaller than that of red blood cells. The duration of the enhancement after bolus injection is currently on the order of several minutes, but enhancement can be prolonged if the USCA is administered using infusion techniques.

The improvement in the microbubble stability achieved during the last decade was done using two principal approaches: external bubble encapsulation with or without surfactants and selection of gases with low diffusion coefficient (such as perfluorocarbon gases). Microbubbles have been stabilized with thin coatings of substances such as palmitic acid or by encapsulatation in microspheres made with albumin, lipids, or polymers. Low-solubility low-diffusibility gases dramatically improve persistence [20]. Most recently developed USCAs combine these two approaches to prolong contrast enhancement. A summary of USCAs and their characteristics is provided in the Table 1.

USCAs can be classified according to their pharmacokinetics [21]. Among the blood pool agents, transpulmonary USCAs offer higher diagnostic potential compared to agents that cannot pass the pulmonary capillary bed after a peripheral intravenous injection. In addition to their vascular phase, some USCAs can exhibit a tissue- or organ-specific phase. Levovist (SHU 508 A, Schering SA, Berlin, Germany) can accumulate within the liver and the spleen for up to 20 min once it has disappeared from the blood pool [22]. This phenomenon may be related to microbubble adherence to the sinusoids. Other USCAs, such as NC100100 (Sonazoid, Nycomed Amersham, Oslo, Norway) or SHU 563A (Sonavist, Schering SA), undergo phagocytosis by the reticulo-endothelial system [23]. Varied types of information can be obtained by applying contrast imaging at different times after the injection, as demonstrated in Fig. 1(b) and (c), which presents images made using Levovist in both the arterial phase and the late organ-specific phase, respectively.

The microbubble ultimately shrinks into the plasma and the dissolved gas is eliminated by the lungs in the expired air while the stabilizing components are eliminated by the liver and kidney. USCA kinetics in the kidney differs from that of the liver due to the absence of any accumulation of the microbubbles in the kidney [24]. 

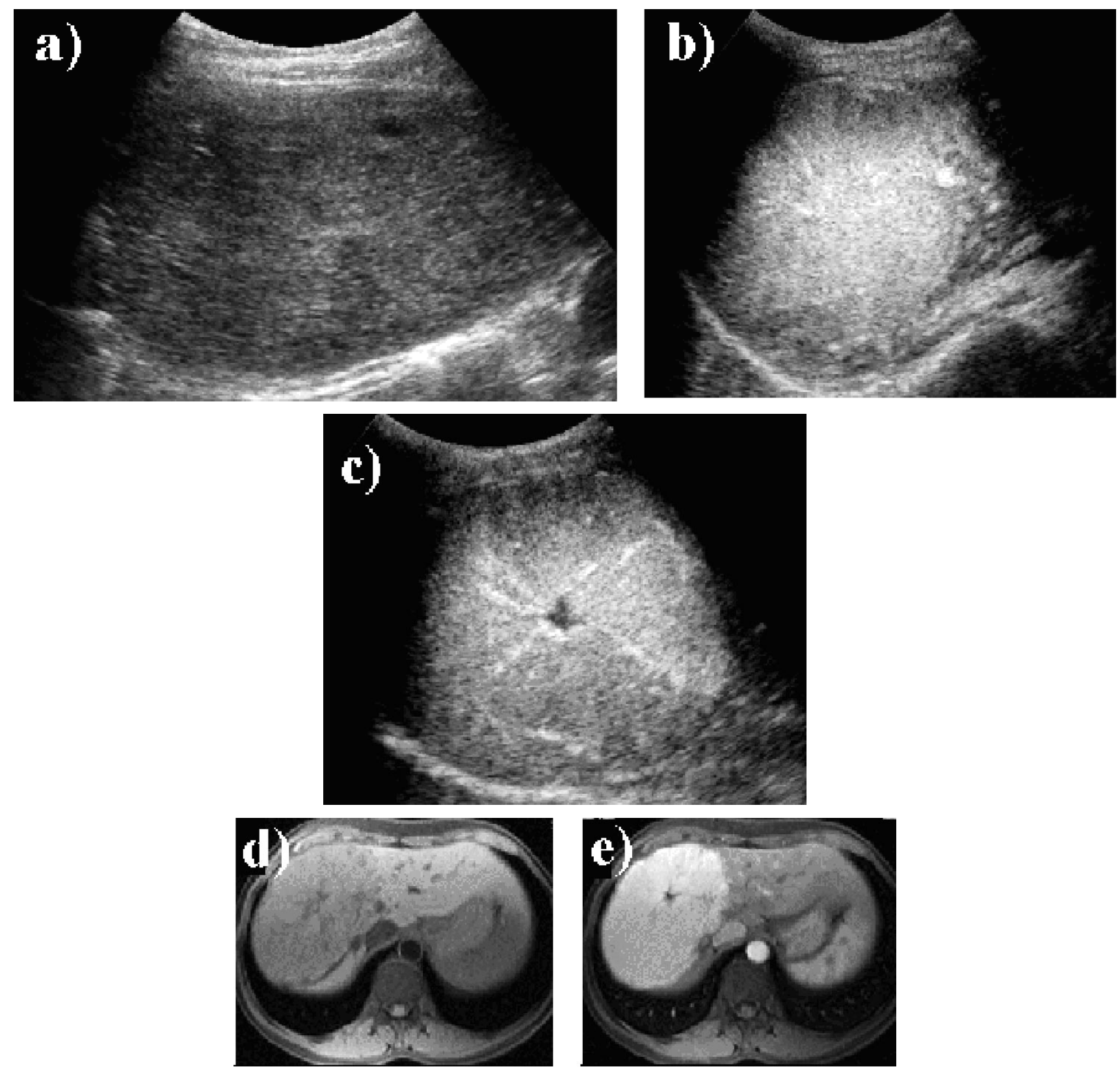

Fig. 1. 40-year-old woman with flank pain. (a)-(c) Ultrasound frames obtained from the upper liver. (d) and (e) MRI correlation obtained at the same level. (a) Transverse B-mode scan of the right liver showing a very large isoechoic lesion occupying segments VII and VIII. (b) Same view using pulse inversion imaging at high MI obtained during the arterial phase after intravenous administration of Levovist. The liver lesion is strongly enhanced. (c) During the late phase scanning (destruction of microbubbles), a central scar with arterial branching vessels is found at the center of the lesion. These ultrasound contrast features are specific of a benign lesion (focal nodular hyperplasia). (d) Baseline dynamic MRI study at the same level showing the lesion with slight hyposignal. (e) Dynamic MRI study, same view obtained during the arterial phase after injection of a Gadolinium chelate MRI contrast agent. The lesion exhibits a strong enhancement and the depiction of the central scar is improved.

\section{Conventional Contrast Imaging}

Preliminary imaging results were obtained with ultrasound systems using conventional modalities such as color and power Doppler or B-mode imaging. Using conventional B-mode, USCAs can opacify large cavities such as the bladder, uterus, cardiac chambers, or some large venous structures. The Doppler modalities, including color, power, and spectral Doppler imaging, are very sensitive to the presence of microbubbles. In cases with failure of nonenhanced Doppler examination, USCAs can be used to increase the intensity of the Doppler signal intensity, but strong artifacts can be produced such as over saturation of the color signals or lateral and axial blooming. (Blooming refers to the artifact observed when color signals overlap the vascular structures and surrounding tissues.) Color and 
power Doppler techniques can be used to map the distribution of the microbubbles using a destruction modality called "stimulated acoustic emission" [22]. Upon applying a high acoustic pressure, the rapid loss of correlation between consecutive echoes resulting from disruption of microbubbles is detected, and a highly contrasted "snap-shot" can be obtained of the microbubble distribution at the instant of destruction [25]. Since the destruction of the microbubbles effectively removes most of them from the imaged region, the rate of imaging must be reduced to allow refilling of the region between acquisitions or, in the case of the liver filled with stored organ-specific contrast, such enhancement can only be obtained once for each selected imaging plane. The major limitation of this technique is the heterogeneity of the enhancement obtained due to regional differences in the microbubble destruction due to the uneven distribution of the acoustic power in the imaging plane.

\section{Development of New Imaging Techniques}

The detection of contrast enhancement closely depends on both the processing of the signals, as well as on the properties of the agent itself. It was the desire to better detect USCA microbubbles that initiated the development of harmonic imaging techniques. As mentioned above, simply acting as very efficient sound scatterers, USCAs were already able to enhance weak Doppler signals and improve the delineation of cardiac and vascular cavities in conventional B-mode images. In fact, microbubbles are very efficient resonant scatterers typically presenting resonant frequencies within the range of medical ultrasound imaging (1-9 MHz). Furthermore, as insonification pressure increases, nonlinear harmonic contributions to the field scattered from the vibrating microbubbles become more and more important [26]. (Up to a certain point, at least, at which the bubble will burst.) It was quickly recognized that even greater contrast between USCA-perfused regions and the surrounding biological tissues could be obtained using the specific nonlinear response of microbubbles.

Nonlinear imaging techniques enhance the visualization of microbubbles within the tissues, and simultaneously reduce the detection of tissue echoes [27], [28]. The presence of the microbubbles in the microvasculature of a tissue increases the echogenicity of the organ. A similar enhancement is observed to that obtained with iodinated contrast agents for CT or Gadolinium compounds for MRI, but with one important difference: USCAs do not permeate to the extravascular space, whereas other types of agents do. Detection of the nonlinear response can be performed using monopulse techniques (conventional harmonic imaging) and subtraction techniques using single (Coherent Imaging Mode, Acuson) or multiple pulses (pulse or phase inversion imaging).

Conventional harmonic imaging was the first nonlinear imaging modality introduced [29]. The second harmonic response is detected at twice the transmit fundamental frequency, using appropriate filters in the frequency domain. The technique was improved recently by optimizing the USCA acoustic response to the transducer and signal processing (Contrast Tuned Imaging, Esaote, Italy). Con- ventional harmonic imaging faces a few limitations. To limit overlap between the transmit frequency bandwidth and the second harmonic signal reception bandwidth, filtering of the system is applied, leading to reduced bandwidth and axial resolution. Furthermore, the higher frequency second harmonic bandwidth is subject to increased attenuation, limiting imaging depth. Nonlinear imaging with subtraction (pulse/phase inversion imaging, pulse subtraction, coherent contrast imaging) relies on the different nonlinear behavior of microbubbles exposed to consecutive pulses of inverted phase with respect to the predominately linear response of biological tissue [27]. Following linear scattering, the echoes from the inverted pulses will still have opposite phases and, thus, their comparison allows to "subtract out" the linear response. Such comparisons between inverted pulses can be performed either temporally (emission of two inverted successive pulses) or spatially (coherent contrast imaging). More than two pulses can be combined to increase sensitivity and reduce motion artifacts (Power Pulse Inversion, ATL, Bothell, WA) [30].

Harmonic imaging was thus developed to exploit nonlinear properties of microbubbles (contrast harmonic imaging), but it was discovered that the same imaging modes could be used to detect the nonlinear properties of biological tissues (tissue or grayscale harmonics). As an ultrasonic wave propagates through tissue, energy at frequencies above the transmit frequency range are gradually generated due to nonlinear propagation [31]. The importance of the effect will increase as the applied acoustic pressure increases. Tissue harmonic imaging has been found to improve ultrasonic imaging quality even without the use of USCAs [32]. A side effect of tissue-harmonic detection is that if better detection of contrast harmonics is sought by increasing the acoustic pressure, the tissue-harmonic response may begin to compete with the USCA response and can actually reduce the level of perceived USCA contrast.

\section{E. Optimized Imaging Sequences}

Microbubble specific imaging sequences have been designed by varying many imaging parameters with a similar approach to that of MRI. Among them, the acoustic power, transmit and receive frequencies, pulse repetition frequency, and triggering of the pulse to the cardiac cycle or an internal clock, pulse phase and amplitude, and number of pulses to build a single image frame are the most critical parameters. These parameters can be combined to design a specific imaging sequences. Using low transmitted acoustic power to limit both USCA destruction and the generation of tissue harmonics, specific pulse sequences taking advantage of microbubble resonant properties currently allow real-time perfusion imaging of several organs, such as the kidney, liver, and myocardium [33]. Such specific pulse sequences require optimization for each USCAs and each clinical indication to obtain the best diagnostic performance [34].

The microbubble behavior within an ultrasonic field depends upon the physical properties of the microbubble population and the local acoustic power [35]. The output power of the ultrasound system is reflected by the mechanical index 

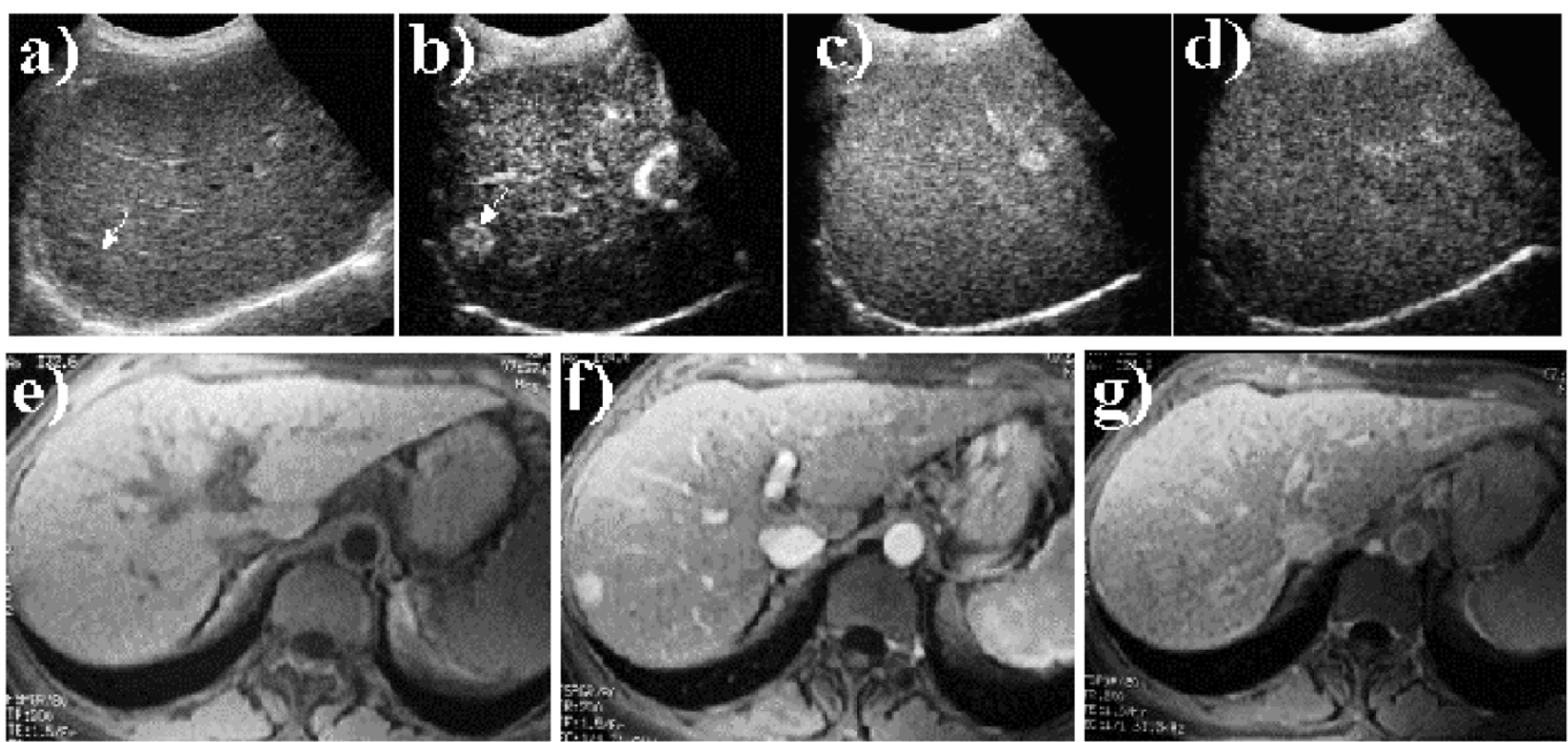

Fig. 2. 72-year-old woman with cirrhosis and hemochromatosis. (a)-(d) Ultrasound frames obtained from the same plane. (e)-(g) Dynamic MRI correlation obtained at the same level. (a) Transverse B-mode scan showing a 2-cm isoechoic lesion of segment VII (arrow) (b) Real-time pulse inversion imaging performed at low MI after a bolus injection of SonoVue, showing arterial enhancement of the lesion (arrow). (c) Same view obtained approximately $30 \mathrm{~s}$ later. The lesion is not visible anymore, as its intrinsic signal decreases and the liver enhances. (d) Same view obtained $2 \mathrm{~min}$ after injection. The lesion becomes hypoechoic to the liver. This sequential enhancement pattern is typical for a small hepatocellular carcinoma and was confirmed by histology. (e) Dynamic MRI sequence obtained before [see (e)] and during arterial [see (f)] and delayed phase [see (g)] following a bolus injection of a Gadolinium chelate MRI contrast agent. The ultrasound images match the MRI pattern.

(MI). Since the microbubble response also depends on the properties of the gas and shell, the MI values given below should not be considered as definite thresholds, but only as relative indications. Four different situations can be considered depending on the microbubble gas (air or perfluorocarbon) and the acoustic power (low or high).

Air-Based USCAs (Such as Levovist): At low acoustic power ( $\mathrm{MI}<0.2$ ), the acoustic response is considered as linear. Microbubbles act as strong scattering objects due to the difference in impedance between air and liquid, and the acoustic response is optimized at the resonant frequency of a microbubble [27].

At higher acoustic power (MI between 0.2-0.5), the microbubbles start to produce a harmonic response at frequencies different from that of the incident wave (fundamental frequency) with subharmonics (half of the fundamental frequency), harmonics (including the second harmonic response at twice the fundamental frequency), and ultra-harmonics obtained at 1.5 or 2.5 times the fundamental frequency [34], [35].

At high acoustic power $(\mathrm{MI}>0.5)$, microbubble destruction begins with emission of high intensity transient signals very rich in nonlinear components. Intermittent imaging becomes needed to allow the capillaries to be refilled with fresh microbubbles. The rate of insonation might be as low as one frame each 2-3 s for renal transplants.

Several images obtained with Levovist and used for the diagnosis of a benign liver lesion are shown in Fig. 1.
Perfluorocarbon Based USCAs (Such as Sonovue): At low acoustic power $(\mathrm{MI}<0.2)$, the microbubble oscillations increase in amplitude and the acoustic response rapidly becomes nonlinear. Since a nonlinear response can be obtained from the microbubbles at relatively low power, these agents present the major advantage that little native tissue harmonic response competes with the strong nonlinear response of the microbubbles [36]. Thus, the contrast between the microbubble and tissue signals is heightened. Tissue perfusion can be studied with multiple sweeps (repeated passage of the imaging plane across the same organ) and real-time (low acoustic power) imaging within a single image plane [37]. However, the attenuation of the ultrasound beam becomes a limitation if the target is deeper than $10 \mathrm{~cm}$. Images from a sequence obtained using real-time pulse inversion imaging of a small hepatocellular carcinoma are presented in Fig. 2.

At higher acoustic power ( $\mathrm{MI}>0.2$ ), the disruption of the microbubbles increases rapidly, as well as the tissue nonlinear response [36]. The microbubbles are detected predominantly in the larger vessels. The access to deeper parenchyma improves.

\section{F. USCA Clinical Indications}

Clinical applications can be divided in four main categories, i.e., Doppler indications, grayscale enhancement of tissue parenchyma and cavities, functional imaging, and therapeutic applications. 
Doppler Indications: USCAs were initially developed to compensate for the lack of sensitivity of Doppler ultrasonography. The Doppler signal intensity is strongly enhanced after an intravenous administration improving the detection and evaluation of vessels in case of low flow, slow flow, deep vessels, or vessels studied with an inadequate angle. Slow administration rates (infusion at $1 \mathrm{ml} / \mathrm{min}$ ) are useful to increase the duration and stability of the enhancement, as well as to limit the blooming artifact [38]. The main Doppler applications of USCAs include renal and liver macrovascular and microvascular diseases (stenosis or occlusion of veins and arteries, infarction, hypoperfusion, focal lesions) [39]-[41]. It might be useful for the detection and characterization of lesions, such as atypical cystic masses. USCAs are particularly useful in case of renal function impairment because of the lack of any renal toxicity. Although the number of Doppler examinations forced to call upon USCA to ensure technical success has been reduced as ultrasound equipment sensitivity has improved, transcranial Doppler imaging also benefits from the strong enhancement of the Doppler signals.

Grayscale Enhancement of Tissue Parenchyma and Cavities: Ultrasound contrast sequences allow detection of parenchymal enhancement depending on the tissue blood flow and volume. These new imaging techniques overcome a major limitation of contrast color Doppler examinations, which is the lack of resolution encountered when the Doppler signals are enhanced. The parenchymal contrast enhancement allows differentiation between cortex and medulla, between renal parenchyma and small tumors, as well as between normally perfused parenchyma and ischemic or infarcted areas. With the introduction of real-time perfusion imaging, the detection of small infarction has become even easier.

USCAs are also helpful for the detection and characterization of renal and liver lesions, as well as for the detection of myocardial perfusion at low MI [42]. The kinetics of the enhancement is similar to CT or MRI, particularly for the arterial phase. USCAs can also be administered into the bladder to diagnose renal reflux [43]. The nonlinear imaging techniques improve the visualization of the microbubbles within the bladder, ureter, and pelvis. The results of contrast enhanced examination were comparable to those obtained by classical radiological tests for the detection or exclusion of the vesico-ureteral reflux.

Newer applications include prostate, breast, and brain imaging. In case of cancer, the nonlinear enhancement can be useful in order to detect and characterize lesions, to guide biopsies, as well as to evaluate the efficacy of treatments [11], [44], [45]. It is also possible to detect brain parenchymal enhancement [46]. This technique might become critical for the assessment of brain infarction.

\section{G. Future Applications}

Future applications include functional imaging combined with quantitative measurements and therapy.

Functional Perfusion Imaging: USCAs can be used as blood pool tracers in a manner similar to that used in nuclear medicine. After a bolus injection of USCA, image time-intensity curves are measured in regions of interest to follow the enhancement. Functional indexes, such as time to peak, peak intensity, wash-in slope, wash-out slope, duration of contrast enhancement, and area under time-intensity curves can be calculated. The additional information provided by functional imaging is currently undergoing evaluation in many areas, such as renal artery stenosis, cirrhosis, and breast lesions [47], [48]. A more recent approach to functional imaging is based upon the destruction of microbubbles at high MI followed by the study of the reperfusion curve observed at low MI. The slope of the reperfusion curve is theoretically related to the blood velocity and the level of enhancement to the local blood volume [42]. These noninvasive measurement techniques have become easier to perform with real-time low acoustic power imaging using perfluorocarbon gas USCAs. Such quantitative assessment of USCAs is becoming critical in the assessment of tumor response to therapy, including angiogenesis inhibitors [49]-[51].

Therapeutic Applications and Microbubble Destruction: Targeted microspheres open new applications for USCAs mediated therapy. They can be charged with active ingredients such as fibrinolytic drugs, nucleotidic chains, and chemotherapeutic drugs [52]. Such compounds could potentially locally deliver active ingredients using ultrasonic energy to break the microbubble carrier. It has also been shown that the ultrasound energy has the potential to increase drug efficacy by sonoporation [53]. The penetration of urokinase within thrombus is increased with the presence of the ultrasonic waves. A similar phenomenon has been reported for gene expression [54]. Investigations of the mechanisms of microbubble destruction should provide a better understanding and ultimately a better control of such processes [55]. Much study is also underway to evaluate potential bioeffects linked to microbubble destruction such as capillary rupturing or cardiac arrhythmias [56], [57].

\section{H. Conclusion}

The development of USCAs is changing the face of conventional ultrasonography. It has become much more than a technique to rescue ultrasound examinations faced with technical limitations. USCAs have led to the development of major new imaging modalities using nonlinear imaging sequences that take advantage of the resonant properties of microbubbles and has provided new techniques for the assessment of parenchymal blood-flow-related enhancement. These microbubble-specific imaging sequences still require optimization by varying parameters such as the acoustic power, transmit and receive frequencies, pulse frequency, pulse phase, and amplitude. Since USCAs are well tolerated and do not have any renal toxicity, contrast enhanced sonography may have one of its most critical clinical impacts in patients with impaired renal function or with strong allergic medical history that preclude the use of iodinated contrast agents. Functional imaging and therapeutic applications are under development and offer very exciting possibilities for the future. 


\section{ULTRASOUND BIOMICROSCOPY}

\section{A. Introduction}

Improved ultrasonic image resolution hinges principally upon reduced wave and pulse lengths and effective focusing of transducers. Significant gains in the resolution of ultrasonic images can be obtained at frequencies above those typically used in medical ultrasound $(>15 \mathrm{MHz})$. The technical innovations introduced using very high-frequency transducers have enabled the ultrasound frequency range from 20 to $100 \mathrm{MHz}$ to be effectively applied to imaging [58]. Imaging at these frequencies enables the visualization of structures with $30-80-\mu \mathrm{m}$ resolution. However, due to higher attenuation at elevated frequencies, the maximum exploration depths are more limited, making high frequencies most suitable for superficial, ocular, intracavitary, and intravascular imaging.

The development of polymer ferroelectric polyvinyldifluoride (PVDF) high-frequency transducers in the late 1980s was a key factor in opening the way for high-frequency ultrasonic imaging. Initial important inroads in the domain of high-frequency ultrasound (ultrasound biomicroscopy) were made in imaging the eye [59], artery wall [60], and skin [61]. Several imaging systems have since been commercialized to target some of the most promising domains for ultrasound biomicroscopy, i.e., dermatology, intravascular, ophthalmology, and small laboratory animal imaging 234 [62].

Existing high-frequency imaging systems generally rely upon rapid mechanical scanning with a single transducer and subsequent reconstruction of image planes from the reflected ultrasonic signals. Thus, although increased frequencies greatly improve axial resolution, the absence of important image-improving technologies that are almost commonplace at lower frequencies, such as transducer arrays and electronic focusing, currently limits imaging quality attainable at higher frequencies. In particular, ultrasound biomicroscopy currently lacks depth-of-field, scanning flexibility, and rapidity relative to its lower frequency counterpart. Recent progress has resulted in commercial arrays operating at a maximum frequency of $20 \mathrm{MHz}$ [63], [64]. Several groups are currently working to confront the acoustic, mechanical, and electronic challenges related to close spacing of highly miniaturized transducer array elements suitable for frequencies beyond $20 \mathrm{MHz}$ [65]-[67]. It is hoped that such work will lead to important future improvements in ultrasonic biomicroscopy.

It of interest to compare the capabilities of ultrasound biomicroscopy with those of optical coherence tomography (OCT) since both technologies provide comparable resolutions and are being developed in parallel. OCT measures back-reflected infrared light instead of acoustical waves.

\footnotetext{
${ }^{1}$ Ultralink LLC, St. Petersburg, FL. [Online]. Available: http://www. arcscan.com

${ }^{2}$ VisualSonics, Toronto, ON, Canada. [Online]. Available: http://www. visualsonics.com/

${ }^{3}$ Boston Scientific, Natick, MA. [Online]. Available: http://www.bostonscientific.com

${ }^{4}$ JOMED, Helsingborg, Sweden [Online]. Available: http://www.jomed. com/products/ivus-us.html
}

Optical as opposed to acoustic differences in material properties serve as the source of reflections, and low-coherence interferometry is used to measure echo-time delays. Images obtained provide a B-mode format cross-sectional view throughout the studied depth of the material with a resolution of 5-20 $\mu \mathrm{m}$. OCT has been used to image the retina through the transparent structures of the eye. It can also provide images of nontransparent tissues such as the artery wall and skin across more limited depths. Comparison with 30-MHz IVUS in saline-filled human coronary arteries demonstrated better delineation of artery layers was obtained with OCT [68]. It should be kept in mind, however, that intavascular probes are improving in terms of imaging quality. Ultrasound also currently holds the advantage for imaging through blood and to more important depths in opaque tissues. Higher sensitivity transducers, allowing the increase of ultrasonic frequencies while maintaining penetration depth, could give a further edge to ultrasound. For very superficial applications, both high-frequency ultrasound and OCT should be considered. Not only competitors, the different natures of the tissue contrast offered by the two techniques could render them complementary for certain applications.

Safety levels for exposure to high-frequency ultrasound merit particular consideration. At any frequency, past a certain power level threshold, ultrasound can potentially cause bioeffects via thermal, mechanical, or cavitation mechanisms. Safety ratings for power levels were established prior to the development of high-frequency transducers. The highly focused nature of high-frequency transducers results in generation of local intensity levels that may exceed that which would be obtained with a more weakly focused lower frequency transducer at the same power level. Furthermore, ultrasonic absorption increases as a function of frequency. Reassuring results have recently been offered showing no thermal damage even for prolonged exposures at $38 \mathrm{MHz}$ of rabbit eye tissues in vivo at intensities well beyond those used in clinical examinations [69]. This is encouraging as corneal and lens tissues are not directly cooled by blood flow and, therefore, should be particularly susceptible to this kind of damage. The effect of the presence of gas bodies and potential bioeffects linked to cavitation should be considered in future high-frequency studies.

\section{B. Fertile Fields in Ultrasound Biomicroscopy}

Dermatology: Dermatosonography with frequencies of over $20 \mathrm{MHz}$ offers a wide range of possibilities in clinical and experimental dermatology. Its application expands to examination of hair follicles, skin tumors, edema, irritant and allergic reactions, scleroderma, psoriasis, wound healing, and skin aging [7]. A series of 20-MHz images of normal skin is presented in Fig. 3. Such images allow rapid in vivo visualization of the highly echogenic collagen-rich dermal layer. The underlying fatty hypodermis contains only isolated echo structure associated with connective tissue bundles. Structures such as hair follicles, vessels, and the subcutaneous portion of nails can also be observed. At $20 \mathrm{MHz}$, the most superficial epidermal structures are only visible at sites such as the heel where the epidermis is 

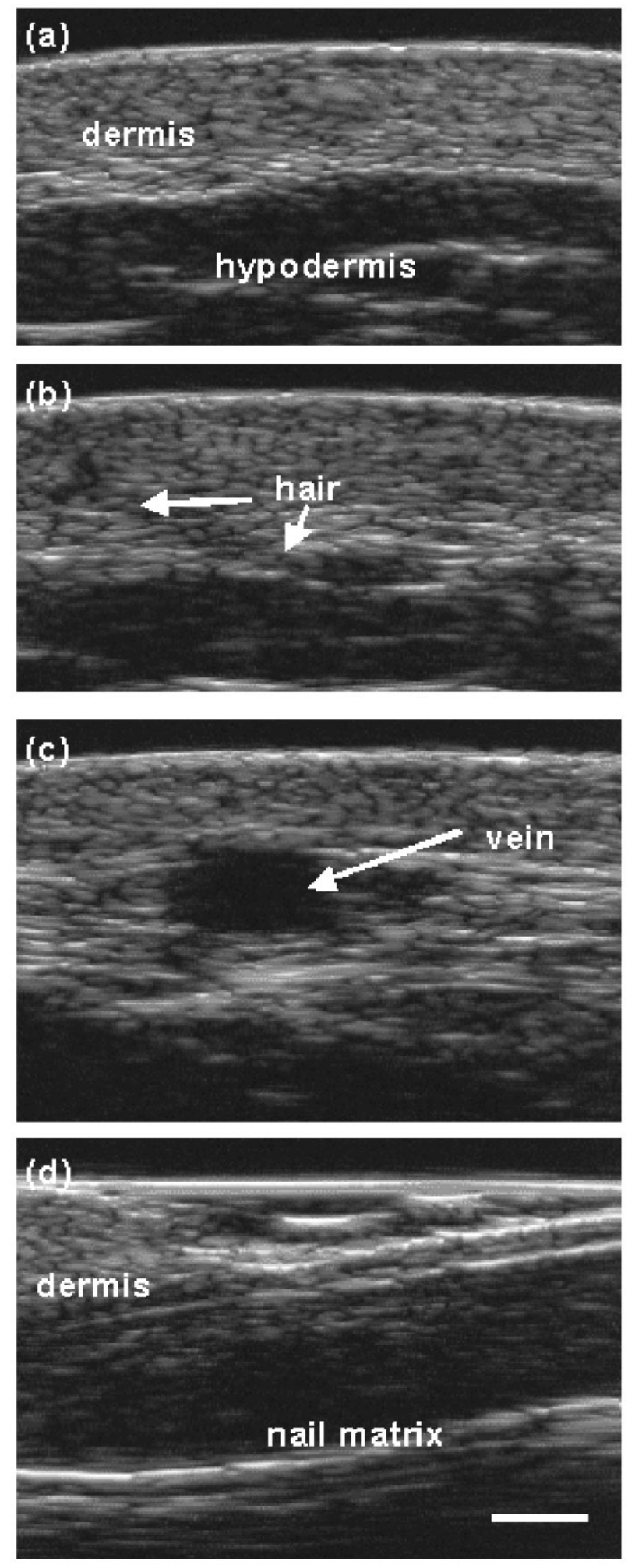

Fig. 3. Images of the skin in vivo obtained with a $20-\mathrm{MHz}$ imaging system (Dermcup 2020, Tours, France). (a) Layers of normal skin. (b) Less echogenic region corresponds to the sub-surface portion of a hair. (c) Vein in cross section. (d) Nail matrix. The white bar denotes $1 \mathrm{~mm}$.

particularly thick or when pathological thickening occurs such as that due to psoriasis. Higher frequency systems (up to $100 \mathrm{MHz}$ ) have been developed specifically for epidermal study [70]. Ultrasonic estimation of the thickness of skin layers provides a simple index that can be linked to modifications as a function of age and hormone replacement therapy [71]. Great interest also exists in the use of such innocuous ultrasonic measurements to lend added credence to claims pertaining to skin age "reversal" with cosmetics.

Ultrasonography of the skin can also be used for evaluation of skin tumors, which are generally recognized as hypoechogenic areas. 2-D or 3-D images are used for preoperative identification of suspect tumors (particularly those which are nonpalpable) for assessment of tumor extent and to scout for surrounding anatomical structures that could complicate surgery [7]. Although some features in ultrasonic images seem to suggest the presence of tumors, no such criteria have yet proven their reliability for diagnosis. After identification of a suspect lesion, dermatological ultrasound may be used to guide biopsy and, thus, obtain a definite diagnosis.

Images of the skin may contain other diagnostic indications. Variation of dermal thickness and echogenicity has been observed with $20-\mathrm{MHz}$ center-frequency ultrasound in vivo and related to changes in dermal hydration [72]. Echogenicity reduction on high-frequency images of the upper dermis has also been proposed as a criterion for evaluating inflammatory skin disease and for monitoring its therapy [73]. Although echogenicity has qualitatively been linked to several clinically interesting processes, specific diagnosis of various skin diseases based on echogenicty is not possible to date.

Intravascular: Percutaneous IVUS offers clinical realtime high-resolution cross-sectional images of the individual layers of the vascular wall. A catheter carrying a miniaturized annular transducer array or a mechanically rotated transducer is navigated through the vascular tree under angiographic guidance until the site of the imaging interest is reached. Initial commercial IVUS systems were limited to the $20-30-\mathrm{MHz}$ range. At these frequencies, penetration depths could be assured on the order of several millimeters (sufficient for imaging across most arterial walls even with a significant plaque burden). However, lateral resolution, which is proportional to the transducer focal length and wavelength and inversely proportional to its diameter, was somewhat disappointing due to the short focal distance and the small active diameter [74]. Currently, commercial IVUS systems propose up to $40-\mathrm{MHz}$ catheter-mounted transducers. This allows some improvement of lateral resolution related to reduced wavelength. Striking images of coronary arteries with thin atherosclerotic plaque have been demonstrated at $60 \mathrm{MHz}$ in vitro [74].

IVUS images allow the assessment not only of the lumen available for blood flow, but also of the layers of the vessel wall, atherosclerotic plaque distribution, or irregularities of the plaque surface (i.e., plaque fissuring). 3-D information can be obtained by acquiring consecutive images during continuous pull back of the catheter. Some information on plaque composition is accessible. Calcified regions of plaque can be identified by strong acoustic reflections with distal shadowing. Some indication of fibrous plaque associated with a regular homogenous pattern of echogenicity and lipid pools 
associated with hypoechogenic regions may also be gleaned. The better resolution provided by newer catheters operating in the $40-\mathrm{MHz}$ range may provide better discrimination of lipid pools [75].

Since IVUS remains minimally invasive, its clinical indications are predominately limited to cases where catheterization is already necessary. In this framework, preintervention use of IVUS to evaluate plaque distribution and to qualitatively evaluate its content can assist in choosing the most promising method for revascularization. Following this, IVUS may be applied to visually monitor the progress of the selected intervention whether it be compression of the plaque by balloon angioplasty or structural restenosis via stent deployment.

Opthalmology: Ultrasound biomicrocopy at $50 \mathrm{MHz}$ can penetrate 4-5 $\mathrm{mm}$ within the eye to image the entire anterior segment in vivo with a resolution of approximately $50 \mu \mathrm{m}$. A mechanically scanned transducer system is positioned on a support and acoustically coupled to the eye across an eye cup filled with couplant [76]. In most patients, the position of the imaged eye can be adjusted by asking the patient to fix his other eye on a visual target. Such visual fixation can be used to suppress eye movement for a brief period.

Ultrasound allows subsurface imaging of the eye even behind opaque eye structures such as the iris and sclera and in patients having visually opaque corneas. Ultrasound biomicroscopy thus provides detailed B-mode images of the cornea, sclera, iris, ciliary body, and anterior lens. Among the many applications of ophthalmologic biomicroscopy are assessment of the angle of the eye in glaucoma and evaluation of the corneal thickness and surface to guide excimer-laser sculpting correction of refractive errors. Definition of the limits of anterior tumors of the eye can provide essential information for preoperative planning of proton irradiation of ocular melanoma [77]. High-frequency ultrasound could also be a useful tool for the evaluation of the biointegration of ocular implants [78].

By using very high frequencies, even finer structural detail can potentially be obtained. For example, images of the rat eye, such as the one presented in Fig. 4 obtained at $80 \mathrm{MHz}$ in vitro, are capable of delimiting epithelial layers as thin as $35-\mu \mathrm{m}$. On this image obtained by a linear lateral displacement of the transducer, it can also be observed that the angle of the beam with respect to the cornea and lens has an important effect on the clarity of the echoes returned from structural interfaces and on the effective penetration depth. To obtain better echo definition toward the angle of the eye, mechanical scanning systems are being developed to follow the curve of the eye in an arc [69], [79].

An additional push has been made to enable 3-D imaging of ocular structures, which is of significant value for defining the anatomy of the angle regions and ciliary body. Systems have been developed allowing acquisition of sequential scans in 3-D so that corneal thickness and angle-to-angle (sulcus-to-sulcus) distance can be measured [80]. The arc-scanning system under development in our laboratory enables real-time 3-D imaging of the entire anterior segment of the eye (sulcus-to-sulcus) in less than $20 \mathrm{~s}$ and is also

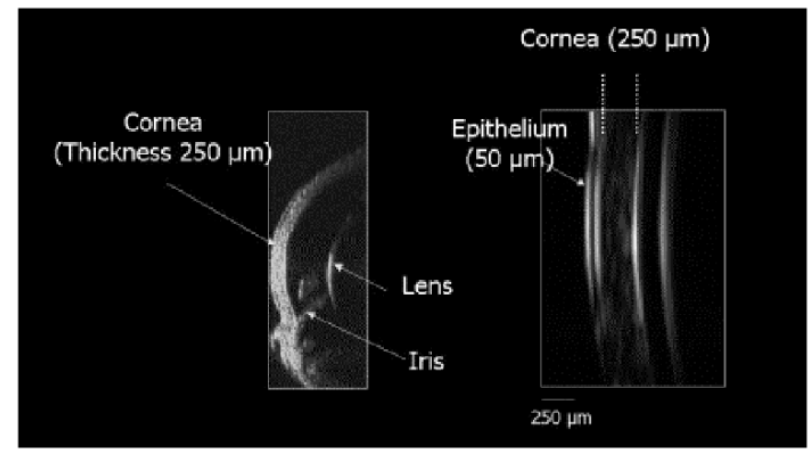

Fig. 4. B-mode image of a rat eye at $80 \mathrm{MHz}$. The structures of the cornea, lens, and iris can be identified. The detailed image on the right-hand side clearly shows the thickness of the corneal epithelium.

suited to high-resolution 3-D exploration of the retina and structures of the posterior wall.

Small Animal Imaging: Bioresearch relies greatly on laboratory mice for models of human pathology, physiology, and genetic research. It is widely recognized that rapid noninvasive screening tools allowing the early identification of physiological or functional anomalies could contribute greatly to studies of genetic and pathological modifications in mice. Furthermore, noninvasive techniques allowing follow-up studies within the same animal would help to reduce the number of animals necessary to obtain statistical significance in a study and would allow continuous evaluation of treatment strategies. Most human genes have a related (homologous) mouse version, making it possible to gain insights into human disease and drug applications. As more drug programs and genetic research utilize mice, the need for small animal imaging is expected to grow dramatically. Among the imaging modalities having the most potential for providing anatomical and functional information in living mice are: 1) MRI; 2) micro CT; 3) micro positron emission tomography; and 4) ultrasonic biomicroscopy.

Currently, ultrasonic investigations of mice are generally made using the highest frequency available probes of clinical systems $(\leq 15 \mathrm{MHz})$. Such systems generally have the advantage of possessing multiple-element arrays providing beam-forming and rapid electronic scanning, but resolution is insufficient for visualization of many structures within the mouse. To best image the mouse, ultrasonic techniques applied for human imaging need to be scaled down by approximately a factor of ten in terms of penetration depths and resolutions. This is consistent with the development of imaging systems in the $20-80-\mathrm{MHz}$ frequency range. A dorsal cross-sectional view of the abdominal cavity of a newborn mouse obtained using a 50-MHz nominal center frequency transducer is presented in Fig. 5. One imaging system for imaging laboratory mice has already been specifically developed by VisualSonics, Toronto, ON, Canada. Recently developed high-frequency Doppler systems also hold considerable interest for vascular studies in the mouse [81].

Among the promising results to date, one can cite the use of $80-\mathrm{MHz}$ imaging in gestating mice with a resolution 


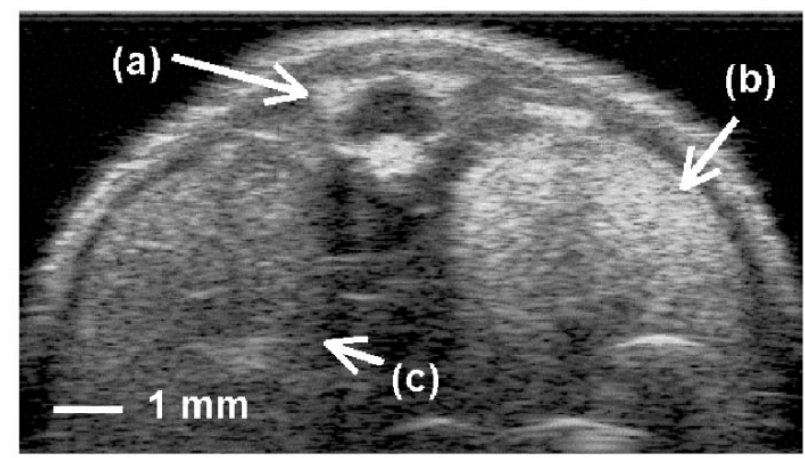

Fig. 5. Cross-sectional dorsal view of the upper abdominal cavity of a newborn mouse (in vitro) obtained using a 50-MHz nominal center frequency transducer. (a) Spinal column. (b) Right Kidney. (c) Liver.

sufficient for the observation of the neural tube and the fetal heart in developing embryos [82]. High-frequency pulsed Doppler measurements have been presented in mouse placenta [83]. Mouse melanoma models can be readily imaged (50-62 MHz) to characterize tumor volume [84], and Doppler signal processing can potentially allow the examination of flow patterns in tumors. One of the remaining challenges for small animal imaging will be to increase its ease of use so that a large number of mice can be screened quickly. Elevated cardiac rates in mice ( $>400$ beats $/ \mathrm{min}$ ) also pose important challenges. With mono-element mechanically scanned systems, this challenge can probably only be met by cardiac synchronization or rapid A-line acquisition along a fixed line-of-site. Greater flexibility in cardiac imaging can be hoped for if higher frequency transducer arrays and rapid electronics continue to develop.

\section{Future Directions}

Quantitative High-Resolution Imaging: Can high frequencies provide quantitative information on tissue composition, as well as high-quality images? The potential exists. Little opportunity (due to lack of appropriately penetrationdepth-scaled acoustic transmission windows) is available for the development of thorough transmission quantitative projection mapping such as used for the evaluation of bone mineral density (BMD). Research for high-frequency QUS has rather focused on analysis of the backscattered signals and construction of B-mode format parameter maps.

Increased device memory and rapid digitization rates have made it possible to capture high-frequency RF signals backscattered from tissue. These RF signals contain information on the phase and frequency content of the signal not available from ultrasonic images. Furthermore, these signals can be obtained free of certain nonlinear compressions and gain stages that are useful for optimization of visual image contrast, but which may mask some of the modifications in the signal due to its interaction with tissue. High-frequency systems generally provide signals with a very large bandwidth, which lend themselves well to spectral analysis techniques. The short transmitted pulse lengths allow very fine segmentation of the backscattered RF data for quantitative analysis.
At high frequencies, the two most-studied types of quantitative indexes have been elastographic deformation and material acoustic properties. Such measurements can potentially provide system-independent and objective information that would add to the information currently available from images alone.

Ultrasound elastography estimates the deformation or the movement of tissues as an external stress is applied. Deformation is usually tracked by applying intercorrelation techniques to segments of RF signals obtained along the same line of site, but as different levels of stress are applied. Elastography techniques are described in detail elsewhere in this issue. It is worth mentioning, however, that as ultrasonic frequencies are increased, the resolution of elastograms increases accordingly. Recent work using 20- and 30-MHz IVUS catheters has been able to present deformation mappings of atherosclerotic plaque with a radial resolution of $200 \mu \mathrm{m}$. In vitro correlations have demonstrated the potential of such mappings to discriminate fibrous, fibro-fatty, and fatty components of atherosclerotic plaque [85]. Similar maps have been produced in vivo using an intravascular probe for the acquisition of RF signals and the natural pressure changes during the cardiac cycle to produce the variable stress [86].

Mappings of the acoustic attenuation and scattering properties of tissues can be obtained using Fourier or parametric spectral analysis of backscattered RF signals coupled with models for the propagation of ultrasound in the medium. Algorithms for estimation of these parameters must also include calibration of the system, usually with respect to a characterized reference phantom or reflecting material, so as to normalize measurements for the system's electromechanical response and ultrasonic beam diffraction. Quantitative information is gained only at the cost of resolution because data must be segmented for signal analysis and spatial averaging is required to reduce variance of parameter estimates. In spite of this, maps of quantitative and system-independent measurements may prove quite useful if they can provide additional diagnostic information.

Spectral estimates, appropriately compensated for the response of the measurement system and for energy losses due to attenuation in tissues between the ultrasound source and the region of measurement, provide information on the frequency-dependent backscatter coefficient of the material (backscatter cross section per unit volume). The frequency dependence of the backscatter coefficient can be linked through models to the size of scattering structures in the tissue relative to the incident wavelength. In a simple, small-scatterer model, the overall amplitude of the backscatter coefficient will be greater if the acoustic contrast of scattering structures relative to the surrounding medium is increased or if their number per unit volume increases [87]. The evolution of spectral content with depth can be linked to tissue attenuation under the assumption that the medium is homogeneous across the studied depth in terms of both scattering properties and propagation velocity. Measurements of both the backscatter and attenuation have been reported in many tissues at frequencies above $20 \mathrm{MHz}$ and related to many diagnostically interesting tissue differences [88]-[90]. 
Ultrasound Contrast Microscopy: USCAs have already considerably increased the imaging and diagnostic capacities of medical imaging in the $2-10-\mathrm{MHz}$ frequency range. Size distributions of existing USCAs were selected to favor resonant behavior in this frequency range. Can contrast techniques also be applied to ultrasound biomicroscopy? Early studies are beginning to respond to this question. Subharmonic and ultraharmonic scattering by current contrast agents (Optison and Definity) has been demonstrated for transmit frequencies up to $26 \mathrm{MHz}$ [91]. Evidence of significant nonlinear propagation and acoustic microbubble destruction was also found [91], [92]. Such findings suggest that it may be possible to apply contrast-specific techniques in conjunction with ultrasound biomicroscpy in the relatively short term.

\section{QuANTITATIVE Ultrasound Bone DENSITOMETRY}

\section{A. Introduction}

The clinical potential of ultrasound for the investigation of pathological conditions that affect bone strength was recognized as early as the 1950s. In 1958, a method was described using measurement of the ultrasonic velocity of a wave propagating along the tibial crest for monitoring fracture healing [93]. Another field of interest in the 1970s was related to the need for a means of monitoring the calcium loss during long-term exposure to weightlessness during manned space flights [94]. Later, a pilot study by Langton et al. in 1984 [95] demonstrated that a group of osteoporotic women could be discriminated from a group of control women using measurements of the ultrasonic frequency-dependent attenuation at low frequency. Since that pioneering study, scientific and clinical interest has been directed at QUS as an alternative method to X-ray absorptiometry for assessing skeletal status. Bone sonometry has become an increasingly popular technique for the diagnosis of osteoporosis, and more than a dozen commercial QUS devices have reached the market [96].

Unlike the majority of medical ultrasound imaging techniques, which use a single transducer or transducer array to transmit and receive ultrasonic signals, most ultrasound techniques that have been developed for the evaluation of bone are based on the transmission of ultrasound from a transmitting source to a receiving source. Such a choice for the measuring configuration is motivated by several factors. Firstly, the transmission approach is analogous to that already used in conventional X-ray to evaluate BMD. Secondly, several sites of measurement such as the heel and wrist lend themselves readily to this type of measurement configuration. The ultasonic image obtained in this fashion is fundamentally different from B-mode images that are generally obtained in ultrasound in that it presents a projection of some property of the traversed material (related, for example, to the time-of-flight or the loss-of-signal amplitude assessed from signals transmitted at different positions).

Osteoporosis has emerged as a major clinical challenge. About one-third of post-menopausal women will have a fracture related to osteoporosis before the end of their lives. If diagnosed sufficiently early, osteoporosis can be treated to significantly reduce the risk of fracture. Moreover, the development of new therapies has heightened interest in monitoring the therapeutic response. Efforts have been made to evaluate factors of fracture risk, the most important of which is bone strength. BMD accounts for most of the strength of bone tissue. Today, reference methods for skeletal status assessment are based on X-ray absorptiometry techniques measuring BMD. However, bone mass is not sufficient to fully characterize bone strength. Several bone properties such as microarchitecture or tissue elasticity, which are not captured by conventional X-ray-based densitometry, also contribute to bone strength independently of bone mass. Quantitative ultrasonic methods may potentially be able to provide information on these additional bone-strength factors.

Bone resorption due to aging and osteoporosis affects cortical and cancellous bone, substantially increasing the fragility of bone. Like X-ray densitometry, QUS techniques have been adapted to assess both cortical and cancellous bone at different skeletal sites. The type of wave propagating, nature of interaction between bone structure and ultrasound, technology, and signal-processing techniques will depend on the skeletal site and the type of bone being investigated. Ultrasound investigations of bone properties uses the transmission of low-frequency ultrasound (from $250 \mathrm{kHz}$ to $1.25 \mathrm{MHz}$, function of the technique and skeletal site) through the bone, including transverse transmission techniques in which the ultrasound wave passes through bone, e.g., heel bone and finger phalanxes, and the so-called axial transmission techniques, in which the ultrasound wave propagates along the long axis of bone, such as the tibia or radius [96]. The speed of sound (SoS, in minutes per second) and/or the slope of the frequency-dependent attenuation (so-called broad-band ultrasonic attenuation (BUA), in decibels per megahertz) of a broad-band ultrasonic pulse transmitted through the skeletal site are calculated [96].

\section{B. Transverse Transmission}

The transverse transmission technique uses two transducers, one transmitter, and one receiver, facing each other on each side of the skeletal site to be tested. The method has been applied to the measurement at the patella, calcaneus, and finger phalanxes. These skeletal sites are easily accessible, with fairly flat and parallel lateral surfaces, thus potentially reducing measurement errors. The calcaneus has been the most extensively studied site. Calcaneal devices show the greatest diversity of technical approaches with fixed single-point systems and imaging devices employing either water bath or ultrasonic gel coupling between the transducers and skin [96]. The following analysis will focus on calcaneus measuring techniques and results.

Ultrasonic measurements at the calcaneus are performed using a classical substitution technique, consisting of the comparison of the Fourier spectrum of the signal transmitted through bone to the spectrum of a reference signal transmitted through a reference medium of known attenuation and SoS (e.g., water) [83]. The frequency variation of the attenuation is nearly linear [97]. The slope of a 
linear regression fit to the frequency-dependent attenuation between $0.2-0.6 \mathrm{MHz}$ yields the BUA value. With most commercial devices, the thickness of measured skeletal site is unknown and, in clinical practice, BUA (in decibels per megahertz) is measured rather than the more conventional slope of attenuation coefficient (in decibels per centimeter megahertz). The influence of soft tissue on BUA is generally considered as being small and is, consequently, neglected.

SoS can be found from the measurement of the timeof-flight of the signal transmitted through bone using specific time criteria or the technique of the phase spectrum. Again, measuring SoS following the above procedure assumes that the impact of soft tissue can be neglected and that the thickness of the skeletal site is known. In practice, there is a great diversity in algorithms from different manufacturers, assuming fixed heel thickness, adjusting for heel thickness, or even introducing soft-tissue correction [96].

Most of the devices, using fixed probes, take measurement in a single point without accurate control of the transducers positioning with respect to the individual heel anatomy. Accuracy and precision are affected by the location within the bone interrogated by the ultrasonic beam. The ultrasound bone imaging system (UBIS) recently developed in our laboratory for the calcaneus has the potential to evaluate standardized regions of interest in all patients and to reproduce the analysis of the same region of bone in a series of scans acquired during long-term follow-up studies. Ultrasound images of BUA and SoS are performed by using a mechanically driven pair of single-element focused transducers immersed in a water bath (Fig. 6) [98]. Chappard et al. recently demonstrated the feasibility of QUS imaging at the wrist with reasonable precision (Fig. 7) [99]. The development of QUS techniques to estimate bone mineral losses during weightlessness of orbital flight, taking into account the constraints applying to space equipment, has led to the development of an imaging devices using waterless contact 2-D transducer arrays [100].

\section{Automatic Region of Interest (ROI)}

The average value of QUS parameters can be calculated by averaging the values at each measurement point in the ROI selected by the operator on the image. The size, form, and location of the ROI is adjustable by the operator. The contribution of the observer to the coefficient of variation is eliminated if an algorithm is utilized that selects the ROI completely automatically. In the calcaneus, a region of lowest attenuation can be found in the greater tuberosity [101]. This property is used by imaging devices to automatically place a circular ROI on images (Fig. 8). Studies using imaging instruments have shown that the use of this automatic ROI improve the in vivo precision compared to the use of fixed ROIs [102].

A problem with the use of this ROI has to be considered. The ROI is circular in shape and of a fixed size, thus imperfectly adapted to the anatomy of each individual. By using a circular ROI, different anatomical locations on the heel will be assessed. Furthermore, long-term changes of the bone structure might shift the position of the ROI, introducing

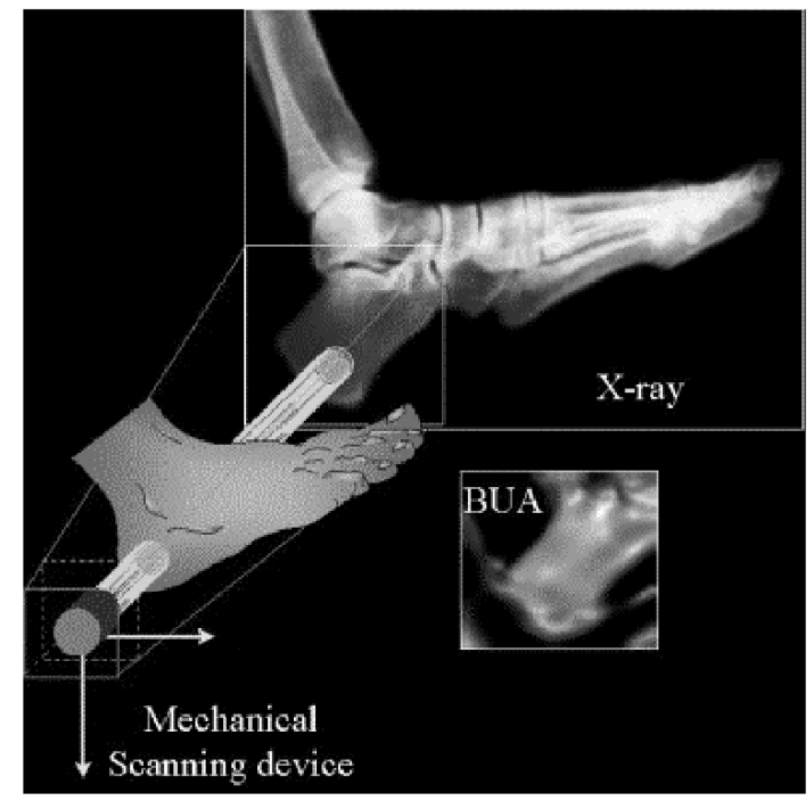

Fig. 6. (bottom) BUA image of the calcaneus and (top) X-ray image of the foot (same subject) obtained by mechanical scanning of the heel immersed in a water bath.

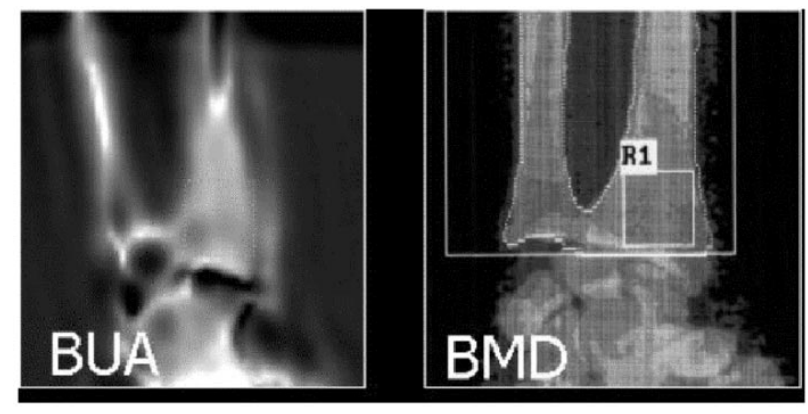

Fig. 7. (left) BUA and (right) BMD image of the wrist (same subject).

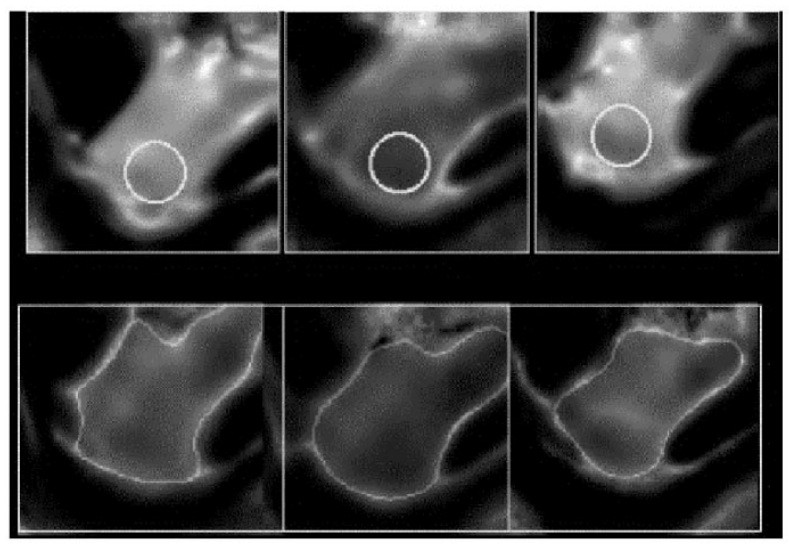

Fig. 8. (top) Automatic circular ROI and (bottom) automatic segmentation of QUS BUA images in three different patients.

errors during monitoring of bone changes. Techniques using anatomical features rather than acoustical features of the calcaneus, as they are known from dual X-ray absorption (DXA), CT, or MRI image processing, might be more robust than algorithms that are based on the inner structure. 


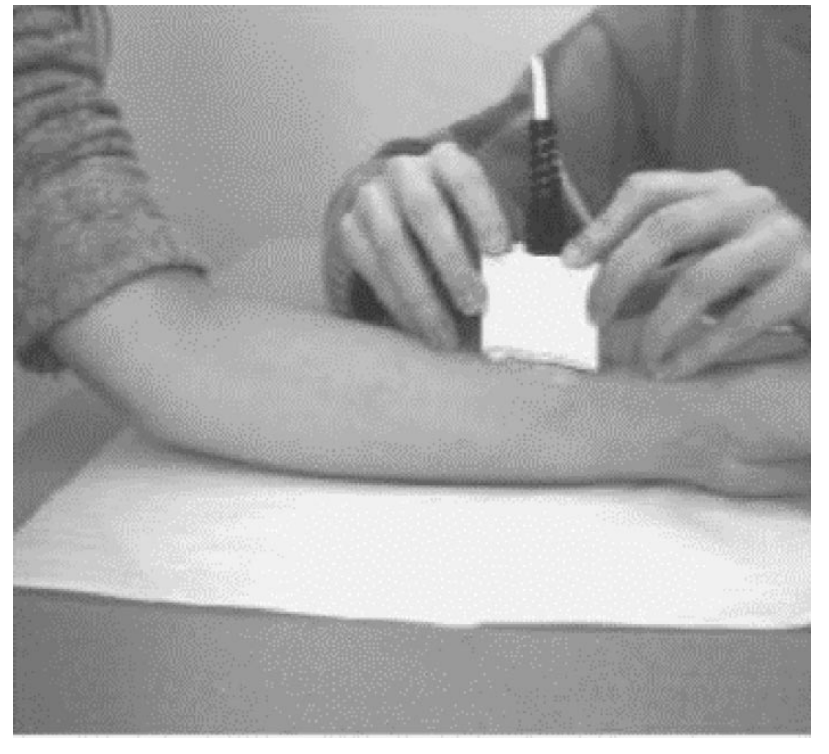

Fig. 9. Ultrasonic probe for multisite axial transmission measurements developed at our laboratory.

Moreover, such algorithms offer better flexibility for defining ROIs including the whole bone. Recently, methods based on elastic deformation of flexible contours (snakes) have been applied to acoustic images of the calcaneus to calculate its outer contour [103] (Fig. 8). The whole calcaneus, as well as different regions inside the bone, can potentially be selected for evaluation by this method.

\section{Axial Transmission}

The axial-transmission technique currently uses a pair (transmitter and receiver) of low-frequency transducers (between $250 \mathrm{kHz}-1 \mathrm{MHz}$, depending on the device) placed on the skin along the bone to measure the velocity of the first arriving signal that has propagated along a fixed distance of the cortical layer of the bone parallel to its long axis. Several measurement sequences combining direct transmission and pulse-echo measurements may be used to assess soft-tissue thickness and correct for soft-tissue errors. The measured velocity corresponds to a wave (so-called lateral wave), which propagates at the longitudinal velocity of bone along the surface of the bone after entering it at the longitudinal critical angle [104]. The transducer-sample and transmitter-receiver distances are chosen such that the lateral wave is the first arriving signal. For a thin plate or cortical shell, dependence of velocity on thickness has been reported [105], suggesting that the lateral longitudinal wave is not consistently the first arriving signal and that other propagating modes, such as plate modes, may arrive first [106].

In contrast to transverse transmission techniques, which require placing a transducer on each side of the bone, the axial transmission technique may be applied to a greater number of skeletal sites (Fig. 9). One device is currently commercially available for the measurement $(1 \mathrm{MHz})$ of several sites, including the radius, finger phalanges, tibia, metatarsal, and hand metacarpal.

\section{E. Clinical Aspects}

Recent reviews confirm QUS diagnostic sensitivity and clinical utility for the prospective assessment of fracture risk [96]. Normal bone demonstrates higher attenuation, and is associated with greater velocity compared with osteoporotic bone. As for X-ray absorptiometry, the influence of age was observed with a more pronounced decrease immediately after menopause. Various retrospective studies have reported the discriminating power of QUS between osteoporotic and age-matched controls to be similar to that of X-ray densitometry techniques [96]. The strongest evidence regarding the association between QUS and fracture risk comes from two large-scale prospective studies of older women [107], [108]. These studies reported similar predictive values for QUS at the calcaneus and X-ray BMD measured at the femur for femoral or nonvertebral fractures in elderly subjects of over 65 years. The association between ultrasonic measurements at the heel and vertebral fracture and QUS measurements at the phalanges and nonvertebral fractures have also been reported [109]. The value of ultrasound densitometry in younger women (i.e., perimenopausal women) is less certain, although the previously cited result was confirmed for a population of younger women (45-75 years) [110]. Finally, there is a strong correlation between BUA and bone density measurements at the same site $\left(r^{2}=0.8\right)$ [111]. This suggests that BUA may be closely linked to bone density at the site of measurement.

There have been limited investigations regarding the ability of QUS to measure bone changes over time. The value of serial measurements, particularly at the calcaneus, to follow response to therapy has been highly controversial. Inconsistent results have been reported between many therapeutic assays. There may be practical reasons for such an inconsistency, i.e.: 1) limited precision of the devices used in clinical trials and 2) insufficient knowledge of factors influencing long-term reproducibility (external and physiological factors) resulting in poorly qualified measurement protocols. Furthermore, it has been argued that measurements from peripheral skeletal sites will not be useful for monitoring since they do not reflect treatment-related bone changes that are observed at central sites, particularly the spine.

In women, so-called primary or idiopathic osteoporosis is the most common type of osteoporosis. A number of studies report on QUS use in other skeletal diseases such as endocrine, renal, or genetic disorders, drug-induced osteoporosis (glucocorticoid), genetic diseases, immobilization, pregnancy and lactation, loco-regional osteoporosis associated with arthritis, and chronic and inherited disorders in childhood [96].

\section{F. Experimental Aspects}

In spite of recent developments [106], [112]-[115], the theoretical complexity to describe the interaction between ultrasound and the complex structure of bone is such that the relationships of QUS parameters and bone properties remain unclear. This has motivated many experimental studies seeking to elucidate the relations between acoustic properties and bone properties. The results have been extensively reviewed in [96]. 
Some of the results, limited to studies performed in human specimens, are summarized here. In the range of densities corresponding to human cancellous bone (calcaneus, vertebra), there is a strong linear relationship $\left(R^{2}: 0.75-0.92\right)$ between acoustic properties and BMD. Bone is acoustically anisotropic. The acoustic anisotropy parallels the structural and mechanical anisotropy. In cancellous bone, BUA and SoS are greatest for a propagation parallel to the predominant trabecular axis. Although there might be some clinically useful information in the ultrasonic characterization of bone anisotropy, this potential benefit has not been thoroughly investigated yet, and the acoustic properties are measured along a single axis in clinical practice, resulting in very strong correlation between ultrasound and BMD. Several studies reported that microarchitecture does not have a strong influence on QUS parameters in unidirectional measurements.

Strong correlations between ultrasonic parameters and mechanical properties (Young's modulus or strength) are reported for femoral and calcaneal specimens. The best prediction of the mechanical properties is obtained with multiple regression models combining SoS and BMD. In contrast, BUA does not provide independent prediction compared to BMD alone. Studies in cadavers compared ultrasonic measurements at the calcaneus with the bone strength of matched calcaneus, femur, or vertebrae. The correlations are strong for the calcaneus, moderate for the femur, and weak, but significant, in the case of the vertebrae. Regarding bone strength, the predictive value of QUS at the calcaneus is comparable or weaker than the prediction obtained with X-ray BMD alone and, again, the combination of the various quantities (BMD and BUA or SoS) does not improve the prediction of strength for the various sites. These results suggest that current QUS measurements may be considered as surrogate for X-ray BMD at the tested skeletal site.

\section{G. Discussion}

Recent interest in bone ultrasound characterization was based on the potential of elastic waves to probe multiple bone properties, including bone density, microarchitecture, and elasticity, in order to completely characterize bone strength. In addition, bone QUS does not involve radiation exposure, and can be easily performed in a primary-care physician's office and, thus, may be suitable for screening large numbers of women for osteoporosis. QUS measurements are predictive of future fracture risk.

For the time being, QUS techniques have mainly been developed for easily accessible peripheral sites, i.e., heel, finger, wrist, or tibia-where the impact of a thin layer of surrounding soft tissue is considered negligible. However, the skeleton is heterogeneous and the pattern of bone loss may vary at different sites depending on age or on the underlying disorder. In addition, if serial monitoring is considered, all skeletal sites will not reflect treatment-related changes equally well. It should also be noted that several bone properties contribute independently to bone strength. Therefore, a site- or parameter-specific measuring technique only provides a partial view of skeletal status.

These considerations guided investigations toward innovative multisite or multiparametric QUS techniques. Innovative reflection techniques such as ultrasound critical reflectometry (UCR) and ultrasonic backscatter have been advanced for the measurement of specific material and structural properties. UCR is a specific technique to measure critical angles at which total reflection occurs for an incident wave impinging on a soft tissue/bone interface. Phase velocity of both compressional and shear waves in bone can be measured using Snell's law [116], [117]. The technique has been developed to measure the variability of velocity along the surface of a bone specimen. Moreover, the velocity can be obtained in many different orientation angles in the plane of the bone surface allowing the principal axis of symmetry to be determined. The UCR technique has been used to characterize cortical bone in vitro and in vivo [118], [119]. The backscatter signal is known to be dependent on the scatterer structure, in this case, the bone microstructure. A few studies have shown the feasibility of the backscatter approach in vitro and in vivo at the calcaneus [120], [121]. The frequency-averaged backscatter coefficient between $0.2-0.6 \mathrm{MHz}$ has been termed the broadband ultrasonic backscatter (BUB). The relationship between the BUB and future fracture risk has been investigated by Roux et al. [122]. These authors reported the ability of the BUB at the calcaneus to discriminate osteoporotic patients from controls. Another interesting approach based on ultrasonic transmission tomography has been explored. The potential of ultrasonic tomography to provide images of attenuation and SoS of bone cross sections was demonstrated in vitro by Seghal et al. [123]. However, bone is a strongly refracting medium compared to surrounding soft tissue and tomographic reconstruction suffers from severe refraction or multipath beam artifacts. To overcome these difficulties, reflection tomography can be combined with transmission tomography to provide more accurate reconstruction of SoS cross-sectional images of bone [124].

Large prospective studies have revealed that ultrasonic parameters measurements at the heel can identify those individuals at risk of osteoporotic fracture as reliably as can dual-energy X-ray absorptiometry. However, several clinical issues need to be addressed as follows.

1) Although many retrospective and prospective studies have consistently reported the predictive value of QUS measurements for fracture risk, there is still no consensus on how to use QUS measurements to manage individual patients. This is probably due to the substantial diversity in QUS approaches (diversity in technological approaches, software and parameters, skeletal sites), which limits comparability among devices and indicates that QUS has not yet reached maturity. However, the potential of widespread use of this technology to assess skeletal status in primitive osteoporosis and many other bone-related diseases is very important.

2) Does combining measurements at several sites enhance fracture risk prediction? Preliminary clinical validation of axial transmission techniques applied to several sites (radius, phalanx, metacarpal, calcaneus) has been reported. Discrimination of osteoporotic fractures at all sites was statistically significant and 
moderate improvement in the discriminative ability was shown from the combination of several sites [125], [126].

3) Does combining several parameters enhance fracture risk prediction? To date, only one study has reported that the combination of backscatter and transmission measurements at the calcaneus did not perform better than either parameter alone [122].

Further clinical studies of multisite or multiparametric approaches are required so that their clinical potential can be fully assessed.

\section{CONCLUSION}

Well-established, widely utilized, and affordable technological medical ultrasound imaging continues to grow and to expand its capabilities. Ultrasound contrast imaging has not only helped improve existing imaging, but has also led to the modification of clinical systems for harmonic signal detection and programming of special imaging sequences. New diagnostic opportunities springing from the development of ultrasound contrast include ultrasonic evaluation of the blood flow in tissues and agent targeting for specific tissue discrimination or for therapeutic vectors. Although not discussed in detail in this paper, 3-D ultrasound is an increasingly important area of ultrasound development. It is a logical evolution of ultrasound technology and complements other parallel developments underway at this time. For example, contrast imaging should promote 3-D ultrasound imaging as 3-D reconstruction of harmonic contrast data could provide vascular anatomy details not available with conventional grayscale, color, and power Doppler. U1trasound biomicroscopy allows a closer ultrasonic look at many tissues such as the eye, skin, and artery wall. It has also been central in allowing ultrasound to compete in the race for the creation of imaging systems adapted for small laboratory animals. Ultrasound already sets itself apart from many other medical imaging modalities due to its real-time and noninvasive nature. The development of ultrasonic bone densitometry has demonstrated that ultrasound can add the label of "quantitative evaluation" to its list of advantages, at least for specific well-defined applications. The work in the domain of bone densitometry can also serve as an example of the steps necessary to evaluate and optimize new diagnostic ultrasound techniques. With such important recent improvements and continued technical advancements on the horizon, the future of ultrasonic imaging can be anticipated to be interesting.

\section{REFERENCES}

[1] T. R. Nelson, D. B. Downey, D. H. Pretorius, and A. Fenster, ThreeDimensional Ultrasound. Philadelphia, PA: Lippincot, Williams \& Wilkins, 1999.

[2] R. L. Dyson, D. H. Pretorius, N. E. Budorick, D. D. Johnson, M. S. Sklansky, C. J. Cantrell, S. Lai, and T. R. Nelson, "Three-dimensional ultrasound in the evaluation of fetal anomalies," Ultrasound Obstet. Gynecol., vol. 16, pp. 321-328, 2000.

[3] J. L. Chin, D. B. Downey, T. L. Elliot, S. Tong, C. A. McLean, M. Fortier, and A. Fenster, "Three dimensional transrectal ultrasound imaging of the prostate: Initial experience with an emerging technology," Can. J. Urol., vol. 6, pp. 720-726, 1999.
[4] H. A. Bogers, J. P. Sedelaar, H. P. Beerlage, J. J. de la Rosette, F. M. Debruyne, H. Wijkstra, and R. G. Aarnink, "Contrast-enhanced three-dimensional power Doppler angiography of the human prostate: Correlation with biopsy outcome," Urology, vol. 54, pp. 97-104, 1999.

[5] O. E. Ofili and N. C. Nanda, "Three-dimensional and four-dimensional echocardiography," Ultrasound Med. Biol., vol. 20, pp. 669-675, 1994.

[6] N. Bruining, C. Lancée, J. R. T. C. Roelandt, and N. Bom, "Threedimensional echocardiography paves the way toward virtual reality," Utrasound Med. Biol., vol. 26, pp. 1065-1074, 2000.

[7] G. B. Jemec, M. Gniadecka, and J. Ulrich, "Ultrasound in dermatology. Part I. High frequency ultrasound," Eur. J. Dermatol., vol. 10, pp. 492-497, 2000.

[8] C. Martinoli, S. Bianchi, M. Dahmane, F. Pugliese, M. P. BianchiZamorani, and M. Valle, "Utrasound of tendons and nerves," Eur. Radiol., vol. 12, pp. 44-55, 2002.

[9] R. S. Adler, "Future and new developments in musculoskeletal ultrasound," Radiol. Clin. North Amer, vol. 37, pp. 623-631, 1999.

[10] R. H. Oyen, "Scrotal ultrasound," Eur. Radiol., vol. 12, pp. 19-34, 2002.

[11] G. J. Rizzatto, "Toward a more sophisticated use of breast ultrasound," Eur. Radiol., vol. 11, pp. 2425-2435, 2001.

[12] N. Grobbelaar and J. A. Bouffard, "Sonography of the knee, a pictorial review," in Seminar Ultrasound CT MR, vol. 21, 2000, pp. 231-274.

[13] C. C. Glüer, "Quantitative ultrasound techniques for the assessment of osteoporosis: Expert agreement on current status. The international quantitative ultrasound consensus group," J. Bone Mineral Res., vol. 12, no. 8, pp. 1280-1288, 1997.

[14] O. Aristizabal, D. A. Christopher, F. S. Foster, and D. H. Turnbull, "40-MHz echocardiography scanner for cardiovascular assessment of mouse embryos," Ultrasound Med. Biol., vol. 24, pp. 1407-1417, 1998.

[15] L. Pourcelot, F. Patat, M. Defontaine, J. M. Gregoire, and M. Berson, "Microgravity," Ultrasound Med. Biol., vol. 26, pp. S144-S146, 2000.

[16] B. B. Goldberg, J. B. Liu, and F. Forsberg, "Ultrasound contrast agents: A review," Ultrasound Med. Biol., vol. 20, pp. 319-333, 1994.

[17] P. S. Epstein and M. S. Plesset, "On the stability of gas bubbles in liquid-gas solutions," J. Chem. Phys., vol. 18, pp. 1505-1509, 1950.

[18] R. F. Mattrey, F. W. Scheible, B. B. Gosink, G. R. Leopold, D. M. Long, and C. B. Higgins, "Perfluorooctylbromide: A liver/spleen-specific and tumor-imaging ultrasound contrast material," Radiology, vol. 145, pp. 759-762, 1982.

[19] K. J. Parker, "A particulate contrast agent with potential for ultrasound imaging of liver," Ultrasound Med. Biol., vol. 13, pp. 555-566, 1987.

[20] S. C. Quay, "Microbubble-based ultrasound contrast agents: The role of gas selection in microbubble persistence," J. Ultrasound Med., vol. 13, pp. S9-S9, 1994.

[21] J. M. Correas, O. Helenon, L. Pourcelot, and J. F. Moreau, "Ultrasound contrast agents. Examples of blood pool agents," Acta Radiol., vol. 412, pp. (S)101-(S)112, 1997.

[22] M. J. Blomley et al., "Stimulated acoustic emission to image a late liver and spleen-specific phase of levovist in normal volunteers and patients with and without liver disease," Ultrasound Med. Biol., vol. 25, pp. 1341-1352, 1999.

[23] C. Marelli, "Preliminary experience with NC100100, a new ultrasound contrast agent for intravenous injection," Eur. Radiol., vol. 9 (Suppl. 3), pp. 343-346, 1999.

[24] J.-M. Correas, A. R. Meuter, E. Singlas, D. R. Kessler, D. Worah, and S. C. Quay, "Human pharmocokinetics of a perfluorocarbon ultrasound contrast agent evaluated with gas chromatography," Ultrasound Med. Biol., vol. 27, pp. 565-570, 2001.

[25] M. J. Blomley et al., "Improved imaging of liver metastases with stimulated acoustic emission in the late phase of enhancement with the US contrast agent SH U 508A: Early experience," Radiology, vol. 210, pp. 409-416, 1999.

[26] P. N. Burns, J. E. Powers, and T. Fritzsch, "Harmonic imaging: A new imaging and Doppler method for contrast-enhanced ultrasound," Radiology, vol. 185(P), p. 142, 1992.

[27] N. de Jong, "Physics of microbubble scattering," in Advances in Echo Imaging Using Contrast Enhancement, N. C. Nanda, Sr. and B. B. Goldberg, Eds. Dordrecht, The Netherlands: Kluwer, 1997, pp. 39-64. 
[28] D. H. Simpson, C. T. Chin, and P. N. Burns, "Pulse inversion Doppler: A new method for detecting nonlinear echoes from microbubble contrast agents," IEEE Trans. Ultrason., Ferroelect., Freq. Contr., vol. 46, pp. 372-382, Mar. 1999.

[29] P. N. Burns, J. E. Powers, D. H. Simpson, V. Uhlendorf, and T. Fritzsch, "Harmonic imaging: Principles and preliminary results," Angiology, vol. 47, pp. S63-S74, 1996.

[30] K. Tiemann et al., "Real-time contrast echo assessment of myocardial perfusion at low emission power: First experimental and clinical results using power pulse inversion imaging," Echocardiography, vol. 16, pp. 799-809, 1999.

[31] E. L. Carstensen and D. R. Bacon, "Biomedical applications," in Nonlinear Acoustics, M. F. Hamilton and D. T. Blackstock, Eds. Chestnut Hill, MA: Academic, 1998, ch. 15, pp. 421-443.

[32] R. S. Shapiro, J. Wagreich, R. B. Parsons, A. Stancato-Pasik, H. C. Yeh, and R. Lao, "Tissue harmonic imaging sonography: Evaluation of image quality compared with conventional sonography," Amer. J. Roentgenol., vol. 171, pp. 1203-1206, 1998.

[33] T. R. Porter, F. Xie, S. Le, A. D'Sa, and P. Rafter, "Increased ultrasound contrast and decreased microbubble destruction rates with triggered ultrasound imaging," J. Amer. Soc. Echocardiogr., vol. 9, pp. 599-605, 1996.

[34] P. J. Frinking, A. Bouakaz, J. Kirkhorn, F. J. Ten Cate, and N. de Jong, "Ultrasound contrast imaging: Current and new potential methods," Ultrasound Med. Biol., vol. 26, pp. 965-975, 2000.

[35] J. E. Powers, P. N. Burns, and J. Souquet, "Imaging instrumentation for ultrasound contrast agents," in Advances in Echo Imaging Using Contrast, N. Nanda, Sr. and B. B. Goldberg, Eds. Dordrecht, The Netherlands: Kluwer, 1997, pp. 139-170.

[36] K. Tiemann, C. Veltmann, A. Ghanem, S. Lohmaier, M. Bruce, S. Kuntz-Hehner, C. Pohl, A. Ehlgen, T. Schlosser, H. Omran, and H. Becher, "The impact of emission power on the destruction of echo contrast agents and on the origin of tissue harmonic signals using power pulse-inversion imaging," Ultrasound Med. Biol., vol. 27, pp. 1525-1533, 2001.

[37] S.-J. Rim, H. Leong-Poi, J. R. Lindner, D. Couture, D. Ellegala, H. Mason, M. Durieux, N. F. Kassel, and S. Kaul, "Quantification of cerebral perfusion with 'real-time' contrast-enhanced ultrasound," Circulation, vol. 104, pp. 2582-2587, 2001.

[38] T. Albrecht et al., "Prolongation and optimization of Doppler enhancement with a microbubble US contrast agent by using continuous infusion: Preliminary experience," Radiology, vol. 207, pp. 339-347, 1998.

[39] A. Bauer, G. Becker, A. Krone, T. Fröhlich, and U. Bogdahn, "Transcranial duplex sonography using ultrasound contrast enhancers," Clin. Radiol., vol. 51 (Suppl. 1), pp. 19-23, 1996.

[40] M. Claudon, P. F. Plouin, G. M. Baxter, T. Rohban, and D. M. Devos, "Renal arteries in patients at risk of renal arterial stenosis: Multicenter evaluation of the echo-enhancer SH U 508A at color and spectral Doppler US Levovist renal artery stenosis study group," Radiology, vol. 214, pp. 739-746, 2000.

[41] M. L. Melany, E. G. Grant, A. J. Duerinckx, T. M. Watts, and B. S. Levine, "Ability of a phase shift US contrast agent to improve imaging of the main renal arteries," Radiology, vol. 205, pp. 147-152, 1997.

[42] K. Wei, A. R. Jayaweera, S. Firoozan, A. Linka, D. M. Skyba, and S. Kaul, "Quantification of myocardial blood flow with ultrasound-induced destruction of microbubbles administered as a constant venous infusion," Circulation, vol. 97, pp. 473-483, 1998.

[43] Darge et al., "Reflux in young patients: Comparison of voiding US of the bladder and retrovesical space with echo enhancement versus voiding cystourethrography for diagnosis," Radiology, vol. 210, pp. 201-207, 1999.

[44] E. J. Halpern, F. Frauscher, M. Rosenberg, and L. G. Gomella, "Directed biopsy during contrast-enhanced sonography of the prostate," Amer. J. Roentgenol., vol. 178, pp. 915-919, 2002.

[45] J. U. Harrer and C. Klotzsch, "Second harmonic imaging of the human brain: The practicability of coronal insonation planes and alternative perfusion parameters," Stroke, vol. 33, pp. 1530-1535, 2002.

[46] G. Seidel, K. Meyer, V. Metzler, D. Toth, M. Vida-Langwasser, and T. Aach, "Human cerebral perfusion analysis with ultrasound contrast agent constant infusion: A pilot study on healthy volunteers," Ultrasound Med. Biol., vol. 28, pp. 183-189, 2002.

[47] J. M. Correas, M. Lafortune, and P. N. Burns, "Detection of renal artery stenosis with B-mode enhancement after administration of a US contrast agent," Radiology, vol. 201 (P), pp. 218-218, 1996.
[48] Y. Hosotani et al., "A new method for evaluation of split renal cortical blood flow with contrast echography," Hypertension Res., vol. 25, pp. 77-83, 2002.

[49] I. Iordanescu, C. Becker, B. Zetter, P. Dunning, and G. A. Taylor, "Tumor vascularity: Evaluation in a murine model with contrast-enhanced color Doppler US effect of angiogenesis inhibitors," Radiology, vol. 222, pp. 460-467, 2002.

[50] R. J. Eckersley, J. P. Sedelaar, M. J. Blomley, H. Wijkstra, N. M. deSouza, D. O. Cosgrove, and J. J. de la Rosette, "Quantitative microbubble enhanced transrectal ultrasound as a tool for monitoring hormonal treatment of prostate carcinoma," Prostate, vol. 51, pp. 256-267, 2002.

[51] F. Forsberg, A. P. Dicker, M. L. Thakur, N. M. Rawool, J. Liu, W. T. Shi, and L. N. Nazarian, "Comparing contrast-enhanced ultrasound to immunohistochemical markers of angiogenesis in a human melanoma xenograft model: Preliminary results," Ultrasound Med. Biol., vol. 28, pp. 445-451, 2002.

[52] Y. Wu et al., "Binding and lysing of blood clots using MRX-408," Invest. Radiol., vol. 33, pp. 880-885, 1998.

[53] K. Tachibana, T. Uchida, K. Ogawa, N. Yamashita, and K. Tamura, "Induction of cell-membrane porosity by ultrasound," Lancet, vol. 353, no. 9162, pp. 1409-1409, 1999.

[54] D. Mukherjee et al., "Ten-fold augmentation of endothelial uptake of vascular endothelial growth factor with ultrasound after systemic administration," J. Amer. Coll. Cardiol., vol. 35, pp. 1678-1686, 2000.

[55] J. E. Chomas, P. Dayton, J. Allen, K. Morgan, and K. W. Ferrara, "Mechanisms of contrast agent destruction," IEEE Trans. Ultrason., Ferroelect., Freq. Contr., vol. 48, pp. 232-248, Jan. 2001.

[56] J. Song, M. Qi, S. Kaul, and R. J. Price, "Stimulation of arteriogenesis in skeletal muscle by microbubble destruction with ultrasound," Circulation, vol. 106, pp. 1550-1555, 2002.

[57] J. F. Zachary, S. A. Hartleben, L. A. Frizzell, and W. D. O'Brien, Jr., "Arrhythmias in rat hearts exposed to pulsed ultrasound after intravenous injection of a contrast agent," J. Ultrasound Med., vol. 21, pp. 1347-1356, 2002.

[58] F. S. Foster, C. J. Pavlin, K. A. Harasiewicz, D. A. Christopher, and D. H. Turnbull, "Advances in ultrasound biomicroscopy," $U l$ trasound Med. Biol., vol. 26, pp. 1-27, 2000.

[59] M. D. Sherar, B. G. Starkoski, W. B. Taylor, and F. S. Foster, "A $100 \mathrm{MHz}$ B-scan ultrasound backscatter microscope," Ultrason. Imaging, vol. 11, pp. 95-105, 1989.

[60] N. Bom and J. Roelandt, Intravascular Ultrasound, Techniques, Developments, Clinical Perspectives. Dordrecht, The Netherlands: Kluwer, 1989.

[61] K. A. Dines, P. W. Sheets, J. A. Brink, C. W. Hanke, K. A. Condra, J. L. Clendenon, S. A. Goss, D. J. Smith, and T. D. Franklin, "High frequency ultrasonic imaging of skin: Experimental results," Ultrason. Imaging, vol. 6, pp. 408-434, 1984.

[62] M. Berson, J. M. Grégoire, F. Gens, J. Rateau, F. Jamet, L. Vaillant, F. Tranquart, and L. Pourcelot, "High frequency $(20 \mathrm{MHz})$ ultrasonic devices: Advantages and applications," Eur. J. Ultrasound, vol. 10, pp. 53-63, 1999.

[63] E. Lacaze, S. Michau, and P. Mauchamp, "20 MHz ultrasound array for medical imaging: From design to image evaluation," in IEEE Ultrasonics Symp., Atlanta, GA, Oct. 7-10, 2001, pp. 1139-1142.

[64] J. Schulze-Clewing, M. J. Eberle, and D. N. Stephens, "Miniaturized circular array," in IEEE Ultrasonics Symp., San Juan, PR, 2000, pp. 1253-1254.

[65] C. E. Morton and G. R. Lockwood, "Design of a $40 \mathrm{MHz}$ annular array," in IEEE Ultrasonics Symp., Atlanta, GA, 2001, pp. $1135-1138$.

[66] J. D. Hamilton and M. O'Donnell, "High frequency ultrasound imaging with optical arrays," IEEE Trans Ultrason., Ferroelect., Freq. Contr., vol. 45, pp. 216-235, Jan. 1998.

[67] T. A. Ritter, T. R. Shrout, and K. K. Shung, "Development of high frequency medical ultrasound arrays," in IEEE Ultrasonics Symp., Atlanta, GA, Oct. 7-10, 2001, pp. 1127-1133.

[68] P. Patwari, N. J. Weissman, S. A. Boppart, C. Jesser, D. Stamper, J. G. Fujimoto, and M. E. Brezinski, "Assessment of coronary plaque with optical coherence tomography and high frequency ultrasound," Amer. J. Cardiol., vol. 85, pp. 641-644, 2000.

[69] R. H. Silverman, F. L. Lizzi, B. G. Ursea, L. Cozzarelli, J. A. Ketterling, C. X. Deng, R. Folberg, and J. Coleme, "Safety levels for exposure of cornea and lens to very high-frequency ultrasound," $J$. Ultrasound Med., vol. 20, pp. 979-986, 2001. 
[70] S. E. Gammal, C. E. Gammal, K. Kaspar, C. Pieck, P. Altmeyer, M. Vogt, and H. Ermert, "Sonography of the skin at $100 \mathrm{MHz}$ enables in vivo visualization of stratum corneum and viable epidermis in palmar skin and psoriatic plaques," J. Investigative Dermatol., vol. 113, pp. 821-829, 1999.

[71] L. Chen, M. Dyson, J. Rymer, P. A. Bolton, and S. R. Young, "The use of high-frequency diagnostic ultrasound to investigate the effect of hormone replacement therapy on skin thickness," Skin Res. Tech., vol. 7, pp. 95-97, 2001.

[72] C. Eisenbeiss, J. Welzel, W. Eichler, and K. Klotz, "Influence of body water distribution on skin thickness: Measurements using high-frequency ultrasound," Br. J. Dermatol., vol. 144, pp. 947-951, 2001.

[73] E. Szymanska, A. Nowicki, K. Miosek, J. Litniewski, M. Lewandowski, W. Secomski, and R. Tymkiewicz, "Skin imaging with high frequency ultrasound-Preliminary results," Eur. J. Ultrasound, vol. 12, pp. 9-16, 2000.

[74] F. S. Foster, D. A. Knapik, J. C. Machado, L. K. Ryan, and S. E. Nissen, "High-frequency intracoronary ultrasound imaging," in Seminar Interventive Cardiol., vol. 2, 1997, pp. 33-41.

[75] F. Prati, E. Arbustini, A. Labellarte, B. D. Bello, L. Sommariva, M. T. Mallus, A. Pagoano, and A. Boccanelli, "Correlation between high frequency intravascular ultrasound and histomorphology in human coronary arteries," Heart, vol. 85, pp. 567-570, 2001.

[76] C. J. Pavlin and F. S. Foster, Ultrasound Biomicroscopy of the Eye. New York: Springer-Verlag, 1995.

[77] C. J. Pavlin, J. A. McWhae, H. D. McGowan, and F. S. Foster, "Ultrasound biomicroscopy of anterior segment tumors," Ophthalmology, vol. 99, pp. 1220-1228, 1992.

[78] D. Lheureux, A. Saied, L. Werner, J.-M. Legeais, and G. Berger, "High-frequency ultrasound characterization of microporous biointegrable polymers in cornea using acoustic parameters," Ultrasonics, vol. 38, pp. 1-8, 2000.

[79] D. Z. Reinstein, R. H. Silverman, T. Raevskiy, G. J. Simoni, H. O. Lloyd, D. J. Najafi, M. J. Rondeau, and D. J. Colemen, "Arcscanning very high-frequency digital ultrasound for 3D pachymetric mapping of the corneal epithelium and stroma in laser in situ keratomileusis," J. Refractive Surgery, vol. 16, pp. 414-430, 2000.

[80] D. J. Coleman, R. H. Silverman, and M. J. Rondeau, "Ophthalmology," Ultrasound Med. Biol., vol. 26, pp. S128-S130, 2000.

[81] D. A. Christopher, B. G. Starkoski, P. N. Burns, and F. S. Foster, "High frequency pulsed Doppler ultrasound system for detecting and mapping blood flow in the microcirculation," Ultrasound Med. Biol., vol. 23, pp. 997-1015, 1997.

[82] F. S. Foster, C. J. Pavlin, G. R. Lockwood, L. K. Ryan, K. Harasiewicz, L. Berube, and A. M. Rauth, "High frequency ultrasound backscatter imaging," in IEEE Ultrasonics Symp., Lake Buena Vista, FL, 1991, pp. 1161-1165.

[83] F. S. Foster, G. Liu, J. Mehi, B. S. Starkoski, L. Adamson, Y. Zhou, K. A. Harasiecwicz, and L. Zan, "High frequency ultrasound imaging: From man to mouse," in IEEE Ultrasonics Symp., Atlanta, GA, Oct. 7-10, 2001, pp. 1633-1638.

[84] D. H. Turnbull, J. A. Ramsay, G. S. Shivji, T. S. Bloomfiele, L. From, D. N. Sauder, and F. S. Foster, "Ultrasound backscatter microscope analysis of mouse melanoma progression," Ultrasound Med. Biol., vol. 22, pp. 845-853, 1996.

[85] C. L. de Korte, G. Pasterkamp, A. F. W. van der Steen, H. A. Woutman, and N. Bom, "Charaterization of plaque components with intravascular ultrasound elastography in human femoral and coronary arteries in vitre," Circulation, vol. 102, pp. 617-623, 2000.

[86] C. L. de Korte, S. G. Carlier, F. Mastick, M. M. Doyley, A. F. W. van der Steen, P. A. Serruys, and N. Bom, "Morphological and mechanical information of coronary arteries obtained with intravascular elastography: Feasibility study in vivo," Eur. Heart J., vol. 23, pp. 405-413, 2002.

[87] F. L. Lizzi, M. Ostromogilsky, E. J. Feleppa, M. C. Rorke, and M. M. Yaremko, "Relationship of ultrasonic spectral parameters to features of tissue microstructure," IEEE Trans Ultrason. Ferroelect. Freq. Contr., vol. UFFC-33, pp. 319-329, 1987.

[88] S. L. Bridal, P. Fornes, P. Bruneval, and G. Berger, "Correlation of ultrasonic attenuation (30 to $50 \mathrm{MHz}$ ) and constituents of atherosclerotic plaque," Ultrasound Med. Biol., vol. 23, pp. 691-703, 1997.

[89] C. S. Hall, C. T. Nguyen, M. J. Scott, G. M. Lanza, and S. A. Wickline, "Delineation of the extracellular determinants of ultrasonic scattering from elastic arteries," Ultrasound Med. Biol., vol. 26, pp. 613-620, 2000.
[90] C. Fournier, S. L. Bridal, G. Berger, and P. Laugier, "Reproducibility of skin characterization with backscattered spectra $(12-25 \mathrm{MHz})$ in healthy subjects," Ultrasound Med. Biol., vol. 27, pp. 603-610, 2001.

[91] D. E. Goertz, S. W. S. Wong, C. T. Chin, E. Cherin, P. N. Burns, and F. S. Foster, "Non-linear scattering from microbubble contrast agents in the 14-40 MHz range," in IEEE Ultrasonics Symp., Atlanta, GA, Oct. 7-10, 2001, pp. 1747-1750.

[92] J. K. Poulsen, E. Cherin, T. van der Steen, and F. S. Foster, "Origin of second harmonic component from high frequency ultrasound," in IEEE Ultrasonics Symp., San Juan, PR, 2000, pp. 503-508.

[93] I. M. Siegel, G. T. Anast, and T. Fields, "The determination of fracture healing by measurement of sound velocity across the fracture site," Surg. Gynecol. Obstet., vol. 107, pp. 327-333, 1958.

[94] J. M. Hoop, "Ultrasonic bone densitometer," U.S. Patent 3847 141, 1974.

[95] C. M. Langton, S. B. Palmer, and S. W. Porter, "The measurement of broadband ultrasonic attenuation in cancellous bone," Eng. Med., vol. 13, pp. 89-91, 1984.

[96] C. Njeh, D. Hans, T. Fuerst, C. C. Glüer, and H. K. Genant, Quantitative ultrasound. Assessment of Osteoporosis and Bone Status. London, U.K.: Martin Dunitz, 1999.

[97] S. Chaffaï, F. Padilla, G. Berger, and P. Laugier, "In vitro measurement of the frequency-dependent attenuation in cancellous bone between 0.2-2.0 MHz," J. Acoust. Soc. Amer., vol. 108, pp. 1281-1289, 2000.

[98] P. Laugier, P. Giat, and G. Berger, "Broadband ultrasonic attenuation imaging: A new imaging technique of the os calcis," Calcification Tissue Int., vol. 58, pp. 326-331, 1996.

[99] C. Chappard, C. Roux, G. Berger, and P. Laugier, "Assessment of QUS imaging at the distal forearm," Osteoporosis Int., vol. 11, p. S33, 2000.

[100] M. A. Gomez, M. Defontaine, B. Giraudeau, E. Camus, L. Colin, P. Laugier, and F. Patat, "In vivo performance of a matrix-based quantitative ultrasound imaging device dedicated to calcaneus investigation," Ultrasound Med. Biol., vol. 28, pp. 1285-1293, 2002.

[101] B. Fournier, C. Chappard, C. Roux, G. Berger, and P. Laugier, "Quantitative ultrasound imaging at the calcaneus using an automatic region of interest," Osteoporosis Int., vol. 7, pp. 363-369, 1997.

[102] H. L. Jorgensen and C. Hassager, "Improved reproducibility of broadband ultrasound attenuation of the os calcis by using a specific region of interest," Bone, vol. 21, pp. 109-112, 1997.

[103] F. Lefebvre, G. Berger, and P. Laugier, "Automatic detection of the boundary of the calcaneus from ultrasound parametric images using an active contour model; clinical assessment," IEEE Trans. Med. Imaging, vol. 17, pp. 45-52, Feb. 1998.

[104] E. Camus, M. Talmant, G. Berger, and P. Laugier, "Analysis of the axial transmission technique for the assessment of skeletal status," J. Acoust. Soc. Amer, vol. 108, pp. 3058-3065, 2000.

[105] C. F. Njeh, D. Hans, C. Wu, E. Kantorovich, M. Sister, T. Fuerst, and H. K. Genant, "An in vitro investigation of the dependence on sample thickness of the speed of sound along the specimen," Med. Eng. Phys., vol. 21, pp. 651-659, 1999.

[106] E. Bossy, M. Talmant, and P. Laugier, "2D simulation of the axial transmission technique on a cortical bone plate," J. Acoust. Soc. Amer, vol. 112, pp. 297-307, 2002.

[107] D. Hans, P. Dargent-Moline, A. M. Schott, J. L. Sebert, C. Cormier, P. O. Kotski, P. D. Delmas, J. M. Pouilles, G. Breart, and P. J. Meunier, "Ultrasonographic heel measurements to predict hip fracture in elderly women: The Epidos prospective study," Lancet, vol. 348, pp. 511-514, 1996.

[108] D. C. Bauer, C. C. Glüer, J. A. Cauley, T. M. Vogt, K. E. Ensrud, H. K. Genant, and D. M. Black, "Broadband ultrasound attenuation predicts fractures strongly and independently of densitometry in older women," Arch. Int. Med., vol. 157, pp. 629-634, 1997.

[109] P. J. Huang, P. D. Ross, A. J. Yates, R. E. Walker, K. Imose, K. Emi, and R. D. Wasnich, "Prediction of fracture risk by radiographic absorptiometry and quantitative ultrasound: A prospective study," Calcification Tissue Int., vol. 63, pp. 380-384, 1998.

[110] P. W. Thomson, J. Taylor, R. Oliver, and A. Fisher, "Quantitative ultrasound (QUS) of the heel predicts wrist and osteoporosis-related fractures in women age 45-75 years," J. Clin. Densitometry, vol. 1, pp. 219-225, 1998. 
[111] C. Chappard, P. Laugier, B. Fournier, C. Roux, and G. Berger, "Assessment of the relationship between broadband ultrasound attenuation and bone mineral density at the calcaneus using BUA imaging and DXA," Osteoporosis Int., vol. 7, pp. 316-322, 1997.

[112] A. Hosokawa and T. Otani, "Ultrasonic wave propagation in bovine cancellous bone," J. Acoust. Soc. Amer., vol. 101, pp. 1-5, 1997.

[113] E. T. Hughes, T. G. Leighton, G. W. Petley, and P. R. White, "Ultrasonic propagation in cancellous bone: A new stratified model," Ultrasound Med. Biol., vol. 25, pp. 811-821, 1999.

[114] K. J. Wear, "Frequency dependence of ultrasonic backscatter from human trabecular bone: Theory and experiment," J. Acoust. Soc. Amer, vol. 106, pp. 3659-3664, 1999.

[115] P. Padilla, F. Peyrin, N. Rossignol, and P. Laugier, "Prediction of amplitude and frequency dependence of the backscatter coefficient in cancellous bone with an autocorrelation model," J. Acoust. Soc. Amer., vol. ??, pp. 1122-1129, 2002.

[116] P. P. Antich, J. A. Anderson, R. B. Ashman, J. E. Dowdey, J. Gonzales, R. Murry, J. E. Zerwekh, and C. Y. C. Pak, "Measurement of mechanical properties of bone material in vitro by ultrasound reflection: Methodology and comparison with ultrasound transmission," J. Bone Mineral Res., vol. 6, pp. 417-426, 1991.

[117] S. S. Mehta and P. Antich, "Measurement of shear-wave velocity by ultrasound critical-angle reflectometry," Ultrasound Med. Biol., vol. 23, pp. 1123-1126, 1997.

[118] P. Antich, C. Y. C. Pak, J. Gonzales, J. A. Anderson, R. B. Ashman, K. Sakhaee, and C. Rubin, "Measurement of intrinsic bone quality in vivo by reflection ultrasound: Correction of impaired quality with slow-release sodium fluoride and calcium citrate," J. Bone Mineral Res., vol. 8, pp. 301-311, 1993.

[119] S. S. Mehta, O. K. Oz, and P. Antich, "Bone elasticity and ultrasound velocity are affected by subtle changes in the organic matrix," $J$. Bone Mineral Res., vol. 13, pp. 114-121, 1998.

[120] V. Roberjot, P. Laugier, P. Droin, and G. Berger, "Measurement of the integrated backscatter coefficient of trabecular bone," in Proc. IEEE Ultrasonics Symp., San Antonio, TX, Nov. 1996, pp. 1123-1126.

[121] K. A. Wear and B. S. Garra, "Assessment of bone density using ultrasonic backscatter," Ultrasound Med. Biol., vol. 24, pp. 689-695, 1998.

[122] C. Roux, V. Roberjot, R. Porcher, S. Kolta, M. Dougados, and P. Laugier, "Ultrasonic backscatter and transmission parameters at the os calcis in post-menopausal osteoporosis," J. Bone Mineral Res., vol. 16, pp. 1353-1362, 2001.

[123] M. Seghal, D. G. Lewallen, R. A. Robb, and J. F. Greenleaf, "Ultrasonic imaging of muskuloskeletal system," News Physiol. Sci., vol. 6, pp. 16-20, 1991.

[124] E. Ouedraogo, P. Lasaygues, J. P. Lefebvre, M. Gindre, M. Talmant, and P. Laugier, "Contrast and velocity ultrasonic tomography of long bones," Ultrason. Imaging, vol. 24, pp. 135-156, 2002.

[125] D. Hans, S. K. Srivastav, C. Singal, R. Barkmann, C. F. Njeh, E. Kantorovich, C. C. Glüer, and H. K. Genant, "Does combining the results from multiple bone sites measured by a new quantitative ultrasound device improve discrimination of hip fracture," J. Bone Mineral Res., vol. 14, pp. 644-651, 1999.

[126] R. Barkmann, E. Kantorovich, C. Singal, D. Hans, H. K. Genant, M. Heller, and C. C. Glüer, "A new method for quantitative ultrasound measurements at multiple skeletal sites," J. Clinical Densitometry, vol. 3, pp. 1-7, 2000.

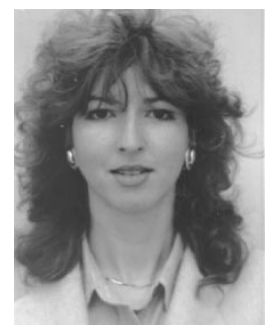

S. Lori Bridal (Member, IEEE) was born in Oklahoma City, OK, in 1967. She received the B.S. degree in physics from Oklahoma State University, Stillwater, in 1988, and the M.A. and Ph.D. degrees in physics from Washington University, St. Louis, MO, in 1990 and 1994, respectively.

In 1995, she joined the Laboratoire d'Imagerie Paramétrique (LIP), University Pierre et Marie Curie, Paris, France. She is currently a Research Scientist with the Centre National de la Recherche Scientifique (CNRS), Paris, France, and continues her research with the LIP on quantitative characterization and imaging of biological materials. Her general research interests include high-frequency ultrasound, tissue characterization, and USCAs.

Dr. Bridal was the recipient of a Chateaubriand Fellowship presented by the French Government for post-doctoral studies.

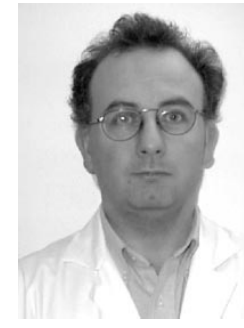

Jean-Michel Correas was born in Oujda, Morocco, in 1962. He received the Radiology degree from the Medical School of Paris, Paris, France, in 1992, the Master's-level degree in medical imaging from the University of Paris (Paris XI), Paris, France, in 1993, and the Ph.D. degree at the University of Tours, Tours, France, in 1999.

From 1994 to 1995, he was a Senior Research Investigator and Manager of Clinical Research with Sonus Pharmaceuticals Inc., Bothell, WA. From 1995 to 1999, he was an Assistant Professor of radiology with the Service de Radiologie Adulte, Hôpital Necker, Paris, France. During this time, he spent one year (1997) as a Visiting Professor of radiology with the Department of Imaging Research, Sunnybrook Health Science Centre, University of Toronto. In parallel, he performed doctoral research. Since 2000, he has been practicing medicine, teaching, and performing research as an Associate Professor of radiology with the Hôpital Necker. His primary fields of interest in clinical practice and research are uroradiology and ultrasound imaging. He is particularly involved in USCA development and applications.

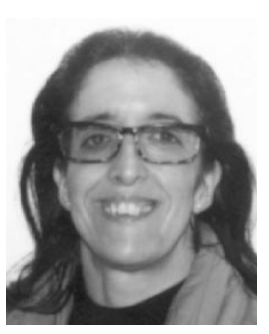

Amena Saïed received the Ph.D. degree in physics from the University of Science and Technology of Montpellier, Montpellier, France, in 1985.

She has been involved for several years in the development of VHF acoustic microscopy $(0.1-2 \mathrm{GHz})$ and specific transducers for nondestructive testing of materials, especially microelectronic devices. In 1990, she joined the Research Department, Schlumberger Industrie, Paris, France, where she was in charge of the development of new gas flowmetering techniques using ultrasound. Since 1992, she has been a Research Scientist with the Laboratoire d'Imagerie Paramétrique, Unité Mixte de Recherche (UMR) 7623, Centre National de la Recherche Scientifique (CNRS), Paris, France. She is the Head of the High Frequency Ultrasound Group. Her research program includes technological innovations in the fields of biomedical imaging and development of new methods of ultrasound signal and image processing for high-resolution and quantitative evaluation of tissue composition and pathologies. She is the Principal Investigator of the Laboratory's projects dealing with the development of 3-D high-frequency $(50-100 \mathrm{MHz})$ quantitative ultrasonography of eye and articular cartilage. Her general research interests include biomedical imaging, tissue characterization, high-frequency transducers, acoustic microscopy, and signal processing.

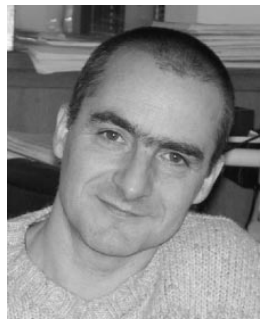

Pascal Laugier is currently a Research Director with the Centre National de la Recherche Scientifique (CNRS), Paris, France, where he heads the Laboratoire d'Imagerie Paramétrique. The Laboratory is comprised of scientists, doctors, and students working in ultrasonic biomedical imaging science. He possesses over 15 years experience in ultrasonic biomedical imaging science. His research has focused on using ultrasound to determine properties of normal and diseased tissues. This approach involves investigations in the field of high-frequency ultrasound and signal- and image-processing techniques. Since the early 1990s, he has led an interdisciplinary group focused on the development and application of tissue characterization techniques to various fields of medicine such as skin, cartilage, eyes, arteries, and bones. A bone ultrasound imaging technique was first patented by he and his coworkers. This technique has been successfully transferred to industry and is now distributed worldwide (UBIS). He serves as an expert reviewer for several national and international institutions. He has authored or coauthored 75 journal papers and 80 conference papers. He holds eight patents, all in the field of ultrasound imaging for medical applications.

Mr. Laugier was the recipient of the 2001 European Grand Prix for Innovation. 